\title{
Sulfonated polyimide/chitosan composite membranes for a vanadium redox flow battery: influence of the sulfonation degree of the sulfonated polyimide
}

\begin{abstract}
Xiaodong Huang, Shuai Zhang, Yaping Zhang, Hongping Zhang and Xuping Yang
We studied the relationship between the sulfonation degree (SD) of the sulfonated polyimide and the properties of sulfonated polyimide/chitosan (SPI/CS) composite membranes for application to a vanadium redox flow battery (VRFB). The SD of SPI was altered from 30 to $70 \%$. The structure and morphology were characterized using attenuated total reflection Fourier transformed infrared spectroscopy (ATR-FTIR) and atomic force microscope, respectively. The physicochemical properties of membranes were also measured. The as-prepared SPI30/CS membrane had the lowest proton conductivity and negligible vanadium ion permeability. The VRFB performance of the SPI30/CS membrane was acceptable and stable at $10 \mathrm{~mA} \mathrm{~cm}^{-2}$, but the chargedischarge process could not be completed at a higher current density, such as 20 and $30 \mathrm{~mA} \mathrm{~cm}^{-2}$, because of its proton conductivity being too low. Although the SPI70/CS membrane had the highest proton conductivity $\left(4.88 \times 10^{-2} \mathrm{~S} \mathrm{~cm}^{-1}\right)$, the coulombic efficiency (CE) of the VRFB containing SPI70/CS was the lowest because of its highest vanadium ion permeability $\left(10.47 \times 10^{-7} \mathrm{~cm}^{2} \mathrm{~min}^{-1}\right)$. In particular, the VRFB assembled with the SPI40/CS membrane underwent 100 charge-discharge cycles at $50 \mathrm{~mA} \mathrm{~cm}^{-2}$ and exhibited good stability in CE (99.3\%) and energy efficiency (EE) (70.5\%). Both SPI40/CS and SPI50/CS membranes are potential candidates for VRFB applications because of their high proton selectivity, good chemical stability and excellent VRFB performance.
\end{abstract}

Polymer Journal (2016) 48, 905-918; doi:10.1038/pj.2016.42; published online 27 April 2016

\section{INTRODUCTION}

The depletion of fossil energy sources, such as coal, oil, and natural gas, has resulted in an increasing demand worldwide for alternative renewable energy sources. However, the output of renewable energy sources, such as solar and wind power, is unstable because of their random and intermittent nature. Therefore, the development of new large energy storage systems is vital for improving the reliability of renewable energy sources. Among several types of energy storage systems including ultra-capacitors, pumped-storage power generation and compressed air, the all-vanadium redox flow battery (VRFB) has attracted increasing interest because of its long cycle life, flexible design, fast response time, deep discharge capability and low cost. ${ }^{1-3}$

The VRFB primarily consists of three types of components including electrolytes, electrodes and anolyte/catholyte separators. A proton-conductive membrane is typically used as the anolyte/catholyte separator to effectively prevent crossover of the catholyte and anolyte and provide a conducting pathway to complete the circuit during the passage of current. An ideal proton-conductive membrane for the VRFB should possess high proton conductivity, low vanadium ion permeability, excellent chemical stability and good mechanical strength and be inexpensive. ${ }^{1-3}$ Currently, perfluorosulfonic membranes, such as Nafion membranes, have been widely applied in VRFBs because of their high proton conductivity and excellent chemical stability. However, Nafion membranes also possess a high vanadium ion permeability and unacceptable costs that limit their large-scale application in VRFBs. ${ }^{4-6}$ As a result, the development of novel proton-conductive membranes with high proton conductivity, low vanadium ion permeability and acceptable costs is important for commercial VRFB applications. Currently, nonfluorinated polymer membranes, such as sulfonated poly(ether ether ketone), ${ }^{7}$ sulfonated polyimide $(\mathrm{SPI})^{8}$ and sulfonated poly(ether sulfone), ${ }^{9}$ have the potential for application to VRFBs. These as-reported nonfluorinated polymer membranes typically exhibit lower vanadium ion permeabilities and costs compared with those of Nafion membranes. However, their chemical stability is also slightly lower than that of Nafion, limiting their long-term VRFB operation.

To improve the chemical stability of nonfluorinated polymer membranes, the commonly used strategies include the preparation of inorganic-organic composite membranes ${ }^{10-13}$ and acid-base composite membranes. ${ }^{14,15}$ In our previous studies, ${ }^{16,17}$ a low-cost acidbase composite membrane (that is, sulfonated polyimide/chitosan (SPI/CS) composite membrane) was prepared using the infiltration and self-assembly method, and this membrane exhibited lower $\mathrm{VO}^{2+}$ permeability and increased chemical stability compared with those of 
the pristine SPI membrane for VRFB applications. However, the effect of the sulfonation degree (SD) of SPI on the performance of the SPI/CS composite membrane remains unknown. This parameter must be optimized to achieve future industrialization of the SPI/CS composite membrane. Therefore, a series of SPI/CS composite membranes with different SDs were prepared in this study. The physicochemical properties and performances of single VRFBs using these SPI/CS composite membranes were investigated.

\section{MATERIALS AND METHODS}

\section{Materials}

1,4,5,8-Naphthalenetetracarboxylic dianhydride (NTDA) was purchased from Beijing Multi. Tech. (Beijing, China). 4,4'-Diamino-biphenyl 2,2'-disulphonic acid (BDSA) was supplied by Quzhou Rainful Chem. Co. (Quzhou, Zhejiang, China). 4,4'-Oxydianiline (ODA) was purchased from Beshine Chem. Tech. (Beijing, China). Vanadyl sulfate $\left(\mathrm{VOSO}_{4}\right)$ was commercially obtained from Shanghai Huating Chem. Plant (Shanghai, China). $m$-Cresol was purchased from Shanghai Kefeng Chem. Reagent (Shanghai, China). Chitosan (CS) with a viscosity average molecular weight of $300 \mathrm{~kg} \mathrm{~mol}^{-1}$ was purchased from Zhejiang Jinke Marine Biochem. Co., Ltd. (Yuhuan, Zhejiang, China). Triethylamine as well as the other reagents were purchased from Chengdu Kelong Chem. Reagent (Chengdu, Sichuan, China). The Nafion 117 membrane was commercially supplied by DuPont Co. (Wilmington, DE, USA).

\section{Preparation of SPI/CS composite membranes}

SPI was synthesized from ODA, BDSA and NTDA in the presence of triethylamine and benzoic acid according to our previously published procedure, ${ }^{16}$ and the SD of SPI was controlled at 30, 40, 50, 60 and $70 \%$ by adjusting the BDSA to ODA ratio, as summarized in Table 1. The SPI (in triethylammonium salt form) membranes with different SDs were obtained by casting their $m$-cresol solution $(8(\mathrm{w} / \mathrm{v}) \%)$ onto dry clean glass sheets followed by drying at $60^{\circ} \mathrm{C}$ for $24 \mathrm{~h}$. Next, these SPI membranes (in triethylammonium salt form), which possessed different SDs, were peeled off the glass sheets and soaked in methanol for $24 \mathrm{~h}$ to remove the residual solvent and/or impurities. Then, the protonization of the SPI membranes (in triethylammonium salt form) was carried out in a $1 \mathrm{moll}^{-1} \mathrm{H}_{2} \mathrm{SO}_{4}$ solution at $25^{\circ} \mathrm{C}$ for $24 \mathrm{~h}$, and the SPI membranes (in proton form) with a thickness of $45 \mu \mathrm{m}$ were thoroughly washed with deionized water and dried at $80^{\circ} \mathrm{C}$. These membranes are referred to as SPI30, SPI40, SPI50, SPI60 and SPI70, respectively. The intrinsic viscosity of SPI30, SPI40, SPI50, SPI60 and SPI70 were measured to be 1.61, 1.52, 1.42, 1.22 and $1.05 \mathrm{dl} \mathrm{g}^{-1}$ (see Table 2), respectively, and the corresponding viscosity average molecular weights ranged from 176.8 to $271.1 \mathrm{~kg} \mathrm{~mol}^{-1}$.

A CS solution $(2(\mathrm{w} / \mathrm{v}) \%)$ was prepared by dissolving CS powder in a 2 $(w / v) \%$ acetic acid solution at $70{ }^{\circ} \mathrm{C}$. After the CS powder was completely dissolved, the CS solution was maintained at $25^{\circ} \mathrm{C}$.

The SPI/CS composite membranes were prepared using the immersion and self-assembly method ${ }^{16}$ as follows: the as-prepared SPI30, SPI40, SPI50, SPI60 and SPI70 membranes were immersed in the CS solution at $25^{\circ} \mathrm{C}$ for $24 \mathrm{~h}$. Then, these SPI/CS composite membranes were removed from the CS solution and dried at $50{ }^{\circ} \mathrm{C}$ for $6 \mathrm{~h}$. Next, these membranes were immersed in $2 \mathrm{~mol}^{-1}$

Table 1 Amount of reagents used to synthesize SPI with different sulfonation degrees

\begin{tabular}{lccccc}
\hline Membrane & Sulfonation degree & BDSA $(g)$ & ODA $(g)$ & NTDA $(g)$ & TEA (ml) \\
\hline SPI30 & $30 \%$ & 1.65 & 2.24 & 4.32 & 3.2 \\
SPI40 & $40 \%$ & 2.20 & 1.92 & 4.32 & 4.2 \\
SPI50 & $50 \%$ & 2.75 & 1.60 & 4.32 & 5.3 \\
SPI60 & $60 \%$ & 3.30 & 1.28 & 4.32 & 6.4 \\
SPI70 & $70 \%$ & 3.86 & 0.96 & 4.32 & 7.4 \\
\hline
\end{tabular}

Abbreviations: BDSA, 4,4'-diamino-biphenyl 2,2'-disulphonic acid; NTDA, 1,4,5,8naphthalenetetracarboxylic dianhydride; ODA, 4,4'-oxydianiline; SPI, sulfonated polyimide; TEA, triethylamine. sulfuric acid for $10 \mathrm{~h}$ to crosslink the CS. Then, these membranes were washed with deionized water and dried at $50^{\circ} \mathrm{C}$ for $12 \mathrm{~h}$ to afford SPI/CS composite membranes with thicknesses of $\sim 50 \mu \mathrm{m}$. The representative scheme for the preparation of the SPI/CS composite membrane is shown in Figure 1.

\section{Characterization of membranes}

Instrumental characterization. The attenuated total reflection Fourier transformed infrared spectroscopy (ATR-FTIR spectra) $\left(4000-660 \mathrm{~cm}^{-1}\right.$, Ge crystal, penetration depth from 2.5 to $25 \mu \mathrm{m}$ depending on the wavelength, resolution $4 \mathrm{~cm}^{-1}$ ) of membranes were recorded using a Nicolet-5700 spectrometer (Thermo Nicolet Co., Madison, WI, USA). The ${ }^{1} \mathrm{H}$ nuclear magnetic resonance $\left({ }^{1} \mathrm{H}\right.$ NMR) spectra were recorded on a Bruker advance III (600 MHz, Karlsruhe, Baden-Wurttemberg, Germany) with DMSO- $\mathrm{d}_{6}$ as the solvent and tetramethylsilane as the internal standard. The morphologies of the membranes were examined using an atomic force microscope (SPI3800N, Seiko Co., Fujimino-shi, Saitama-Ken, Japan) after the membrane samples were dried at $60{ }^{\circ} \mathrm{C}$ for $10 \mathrm{~h} .{ }^{18-20}$ The cross-section of the membranes was observed using scanning electron microscope (Ultra 55, Zeiss Instrument Co., Berlin, Germany) with an acceleration voltage of $15 \mathrm{kV}$, and the cross-section of the membranes was obtained using the freeze-fracture technique in liquid nitrogen. Digital photos of the membranes were recorded with a digital camera (Hongmi Note, Xiaomi Co., Beijing, China).

Measurement of the physicochemical properties. The weight percent of CS with respect to SPI in the SPI/CS composite membrane was calculated using Equation (1):

$$
\text { CS content }(\%)=\frac{m^{\prime}-m}{m} \times 100
$$

where $m$ and $m^{\prime}$ are the dry weights of the pristine SPI membrane and the SPI/CS composite membrane ( $\mathrm{g}$ ), respectively.

The water uptake $(W U)$ is defined as the ratio of the weight of absorbed water to that of a dry membrane, determined as follows: the membrane was immersed in deionized water for $24 \mathrm{~h}$ until completely swollen. Then, the membrane was removed, and the surface moisture was wiped off with a tissue paper. Next, the membrane was quickly weighed. The wet membrane was dried at $60^{\circ} \mathrm{C}$ for $10 \mathrm{~h}$ and then weighed again. The $W U$ was calculated according to Equation (2):

$$
W U(\%)=\frac{W_{\text {wet }}-W_{\text {dry }}}{W_{\text {dry }}} \times 100
$$

where $W_{\text {wet }}$ and $W_{\text {dry }}$ are the weights of the wet and dry membranes (g), respectively.

The swelling ratio $(S W)$ was determined by the dimensional change as a linear expansion ratio. The SW was calculated using Equation (3):

$$
S W(\%)=\frac{L_{\text {wet }}-L_{\mathrm{dry}}}{L_{\mathrm{dry}}} \times 100
$$

where $L_{\mathrm{dry}}$ and $L_{\text {wet }}$ are the thicknesses of the dry and wet membranes $(\mu \mathrm{m})$, respectively.

The density of the dry membrane $\left(\rho, \mathrm{g} \mathrm{cm}^{-3}\right)$ was measured as follows: first, the membrane was dried at $60^{\circ} \mathrm{C}$ for $10 \mathrm{~h}$ followed by cooling to $20^{\circ} \mathrm{C}$ in a vacuum oven. Next, the weight of the dried membrane $\left(m_{\text {dry }}, \mathrm{g}\right)$ was recorded.

\begin{tabular}{|c|c|c|c|}
\hline Membrane & Sulfonation degree & {$[\eta]\left(d l g^{-1}\right)$} & $M_{\eta}\left(\mathrm{g} \mathrm{mol}^{-1}\right)$ \\
\hline SPI30 & $30 \%$ & 1.61 & 271108 \\
\hline SPI40 & $40 \%$ & 1.52 & 255953 \\
\hline SPI50 & $50 \%$ & 1.42 & 239114 \\
\hline SPI60 & $60 \%$ & 1.22 & 205436 \\
\hline SPI70 & $70 \%$ & 1.05 & 176809 \\
\hline
\end{tabular}
Then, the pycnometer $(10 \mathrm{ml})$ was filled with $n$-hexane, and its weight $\left(m_{\mathrm{r}}, \mathrm{g}\right)$

Table $2[\eta]$ and $M_{\eta}$ of SPI with different sulfonation degrees

Abbreviations: $\mathrm{M}_{\eta}$, viscosity average molecular weight; $[\eta]$, intrinsic viscosities; SPI, sulfonated polyimide. 


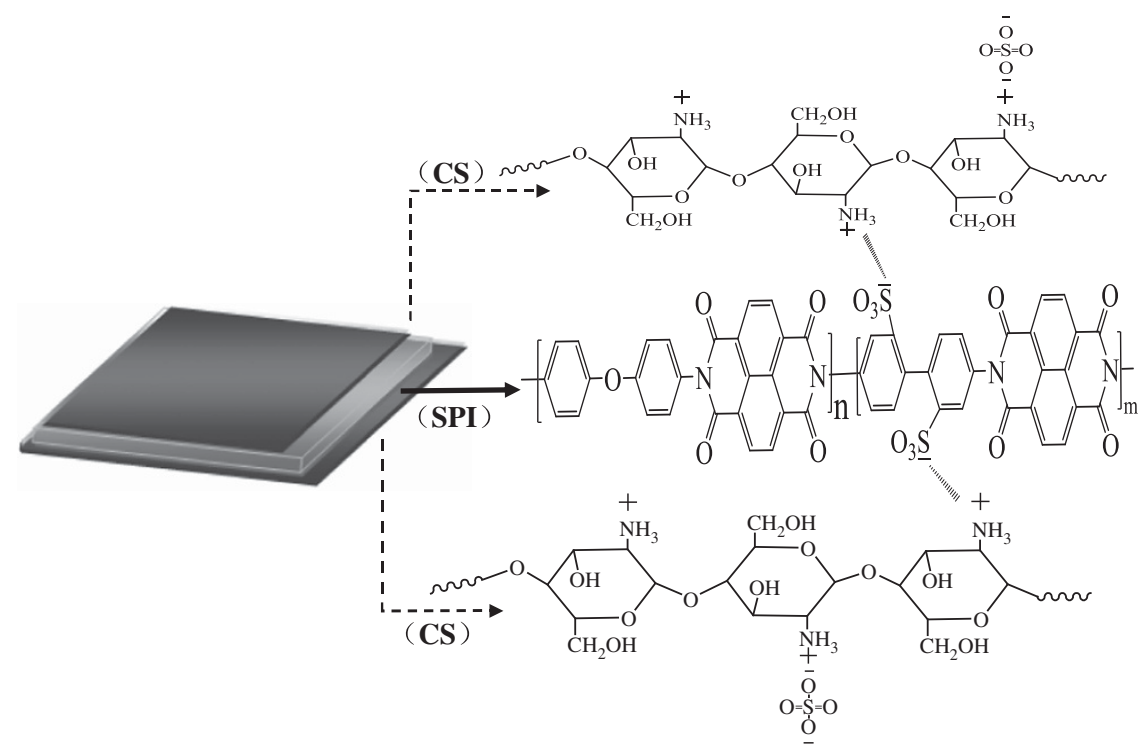

Figure 1 Schematic representation of the sulfonated polyimide/chitosan (SPI/CS) composite membrane. A full color version of this figure is available at Polymer Journal online.

was recorded. Finally, the dry membrane was placed into the pycnometer filled with $n$-hexane, and the total weight of the pycnometer with the membrane and $n$-hexane $\left(m_{\text {total }}, \mathrm{g}\right)$ was recorded. The density of the dry membrane $\left(\rho, \mathrm{g} \mathrm{cm}^{-3}\right)$ was calculated according to Equation (4):

$$
\rho=\frac{m_{\text {dry }}}{m_{\mathrm{r}}+m_{\text {dry }}-m_{\text {total }}} \rho_{\mathrm{r}}
$$

where $\rho_{\mathrm{r}}$ is the density of pure $n$-hexane at $20{ }^{\circ} \mathrm{C}\left(0.692 \mathrm{~g} \mathrm{~cm}^{-3}\right)$.

The ion exchange capacity (IEC) was measured using a conventional titration method. A dry membrane was weighed and immersed into a $1.0 \mathrm{~mol}^{-1} \mathrm{NaCl}$ solution for $24 \mathrm{~h}$ to exchange protons with sodium ions. Then, the membrane was removed and washed with deionized water, and the remaining solution was titrated using a $0.05 \mathrm{moll}^{-1} \mathrm{NaOH}$ solution with phenolphthalein as the indicator. The IEC was determined according to Equation (5):

$$
I E C=\frac{C_{\mathrm{NaOH}} \times V_{\mathrm{NaOH}}}{W_{\mathrm{dry}}}
$$

where $C_{\mathrm{NaOH}}$ and $V_{\mathrm{NaOH}}$ are the concentration $\left(\mathrm{mol} \mathrm{l}^{-1}\right)$ and volume $(\mathrm{ml})$, respectively, of the consumed $\mathrm{NaOH}$ solution.

The chemical stability was measured using a method similar to that previously reported. ${ }^{21-24}$ Briefly, the membrane $(1 \mathrm{~cm} \times 4 \mathrm{~cm} \times 50 \mu \mathrm{m})$ was immersed in $30 \mathrm{ml}$ of $0.1 \mathrm{moll}^{-1} \mathrm{VO}_{2}^{+}+3.0 \mathrm{moll}^{-1} \mathrm{H}_{2} \mathrm{SO}_{4}$ at $40^{\circ} \mathrm{C}$. Then, the integrity of the membranes was examined after 15 days. In addition, the chemical stability of the membrane was also tested in Fenton's reagent (3 wt. \% $\mathrm{H}_{2} \mathrm{O}_{2}+2$ p.p.m. $\mathrm{FeSO}_{4}$ ) at $80{ }^{\circ} \mathrm{C}$. The time required for the membrane to start to break was recorded. ${ }^{25}$

The mechanical properties of the membranes were measured using an electronic universal testing machine (QJ-210A, Shanghai Instrument Co., Ltd, Shanghai, China) using a tensile speed of $10 \mathrm{~mm} \mathrm{~min}^{-1}$ at $20^{\circ} \mathrm{C}$. Before the measurements, after being immersed in deionized water for $24 \mathrm{~h}$ at $20^{\circ} \mathrm{C}$, the wet membranes were cut into dumbbell shapes with the narrowest regions having a width of $10 \mathrm{~mm}$.

Proton conductivity and $\mathrm{VO}^{2+}$ permeability. The proton conductivity was determined using four-point-probe electrochemical impedance spectroscopy. A membrane was rinsed in deionized water for $24 \mathrm{~h}$ before the measurement, and then the membrane was fixed in a measurement cell that was made from Teflon and consisted of two stainless steel flat outer current-carrying electrodes and two platinum wire inner potential-sensing electrodes. The membrane, which was $1 \mathrm{~cm}$ wide and $4 \mathrm{~cm}$ long, was mounted on the cell. The impedance was measured using a CHI760C electrochemical workstation (Shanghai
Chenhua Instruments Co., Shanghai, China) in galvanostatic mode with an AC current amplitude of $5 \mathrm{~mA}$ over a frequency range of $1.0 \mathrm{~Hz}$ to $100 \mathrm{kHz}$ at room temperature $\left(25^{\circ} \mathrm{C}\right)$. The proton conductivity was calculated using Equation (6):

$$
\sigma=\frac{L}{R \times S \times d}
$$

where $\sigma$ is the proton conductivity $\left(\mathrm{S} \mathrm{cm}^{-1}\right), R$ is the membrane resistance $(\Omega), L$ is the distance between the two platinum wires $(\mathrm{cm})$ and $S$ and $d$ are the width $(\mathrm{cm})$ and thickness $\left(10^{-4} \mathrm{~cm}\right)$, respectively, of the membrane.

The vanadium ion permeability across the membrane was measured using a diffusion cell that was composed of two identical compartments with a membrane as the separator. The left compartment was filled with $120 \mathrm{ml}$ of $1.0 \mathrm{moll}^{-1} \mathrm{VOSO}_{4}$ in a $2.0 \mathrm{~mol} \mathrm{l}^{-1} \mathrm{H}_{2} \mathrm{SO}_{4}$ solution, and the right one was filled with $120 \mathrm{ml}$ of $1.0 \mathrm{moll}^{-1} \mathrm{MgSO}_{4}$ in a $2.0 \mathrm{moll}^{-1} \mathrm{H}_{2} \mathrm{SO}_{4}$ solution. $\mathrm{MgSO}_{4}$ was used to equalize the ionic strength of the two solutions and reduce the osmotic pressure effect. The effective membrane area was $5.31 \mathrm{~cm}^{2}$. The sample solution was removed from the right compartment at regular time intervals, and the concentration of $\mathrm{VO}^{2+}$ was determined using ultravioletvisible spectroscopy (UV-1100, MAPADA, Shanghai, China). The $\mathrm{VO}^{2+}$ concentration in the left compartment changed very little during the entire experiment and is considered negligible. Therefore, the $\mathrm{VO}^{2+}$ concentration in the right compartment as a function of time can be expressed as shown in Equation (7):

$$
\frac{\mathrm{d} C_{\mathrm{R}(t)}}{\mathrm{d} t}=\frac{A \times P}{V_{\mathrm{R}} \times d}\left[C_{\mathrm{L}}-C_{\mathrm{R}(t)}\right]
$$

where $C_{\mathrm{L}}$ is the $\mathrm{VO}^{2+}$ concentration in the left compartment $\left(\mathrm{moll}^{-1}\right)$ and $C_{\mathrm{R}(t)}$ is the $\mathrm{VO}^{2+}$ concentration $\left(\mathrm{moll}^{-1}\right)$ in the right compartment at permeation time $t(\mathrm{~min}) . V_{\mathrm{R}}$ is the volume of the right compartment $(\mathrm{ml})$. $A\left(\mathrm{~cm}^{2}\right)$ and $d\left(\times 10^{-4} \mathrm{~cm}\right)$ are the area and thickness of the membrane, respectively. $P$ is the $\mathrm{VO}^{2+}$ permeability coefficient of the membrane $\left(\mathrm{cm}^{2} \mathrm{~min}^{-1}\right)$. In addition, we assume that $P$ is independent of the vanadium ion concentration.

Single VRFB tests. The charge--discharge test of a single VRFB was conducted at $25{ }^{\circ} \mathrm{C}$ using a procedure that was similar to a previously reported protocol $^{8,10-12}$ in which the membrane was sandwiched between the positive and negative half-cell. Figure 2 shows a schematic representation of our single VRFB testing apparatus, where the graphite polar plate, Teflon frame, graphite felt electrode, membrane, graphite felt electrode, Teflon frame and graphite polar plate were compacted from left to right. The effective area of the 
membrane was $8 \mathrm{~cm}^{2}$. The electrolytes consisted of $30 \mathrm{ml}$ of $1.5 \mathrm{~mol} \mathrm{l}^{-1} \mathrm{~V}^{3+}$ in a $3.0 \mathrm{moll}^{-1} \mathrm{H}_{2} \mathrm{SO}_{4}$ solution as the negative electrolyte and $30 \mathrm{ml}$ of $1.5 \mathrm{~mol} \mathrm{l}^{-1} \mathrm{VO}^{2+}$ in a $3.0 \mathrm{~mol} \mathrm{l}^{-1} \mathrm{H}_{2} \mathrm{SO}_{4}$ solution as the positive electrolyte. During the test, the electrolytes were cycled through the half-cell using peristaltic pumps (BT-100SD, Shanghai Qingpu Huxi Instrument Factory, Shanghai, China) at a flow rate of $40 \mathrm{ml} \mathrm{min}{ }^{-1} \cdot{ }^{26}$ The single VRFB chargedischarge test was carried out via a constant current model with a current density that ranged from 10 to $80 \mathrm{~mA} \mathrm{~cm}^{-2}$ using a CT-3008-5 V/3A-S1 battery testing system (Xinwei NEWARE, Shenzhen, Guangdong, China).

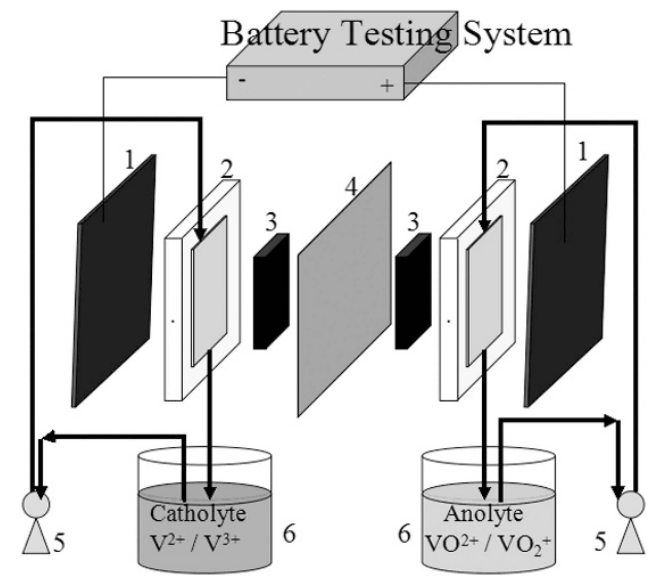

Figure 2 Schematic representation of the single vanadium redox flow battery (VRFB) testing apparatus: 1, graphite polar plate; 2, Teflon frame; 3, graphite felt electrode; 4, membrane; 5, peristaltic pump; 6 , electrolyte tank. A full color version of this figure is available at Polymer Journal online.
To avoid corrosion of the graphite felt electrode and graphite polar plates, the upper limit voltage for charge and the lower limit voltage for discharge were 1.65 and $0.8 \mathrm{~V}$, respectively. The coulombic efficiency $(C E)$, voltage efficiency $(V E)$ and energy efficiency $(E E)$ of the single battery were calculated using Equations $(8,9,10)$ :

$$
\begin{aligned}
& C E(\%)=\frac{C_{\mathrm{dis}}}{C_{\mathrm{ch}}} \times 100 \\
& E E(\%)=\frac{E_{\mathrm{dis}}}{E_{\mathrm{ch}}} \times 100 \\
& \operatorname{VE}(\%)=\frac{E E}{C E} \times 100
\end{aligned}
$$

where $C_{\text {dis }}$ is the discharge capacity $(\mathrm{mAh})$ and $C_{\mathrm{ch}}$ is the charge capacity $(\mathrm{mA} \mathrm{h})$ of the VRFB. $E_{\text {dis }}$ is the discharge electric energy $(\mathrm{mW} \mathrm{h})$ and $E_{\mathrm{ch}}$ is the charge electric energy ( $\mathrm{mW} \mathrm{h}$ ) of the VRFB.

\section{RESULTS AND DISCUSSION}

ATR-FTIR and ${ }^{1} \mathrm{H}$ NMR analysis

The chemical structures of pristine SPI membranes with different SDs were confirmed by their ATR-FTIR spectra shown in Figure 3a. The disappearance of the peak at $1780 \mathrm{~cm}^{-1}$ confirmed the complete imidization of SPI, as reported by Gills et al. ${ }^{27}$ The peaks at 1712 and $1670 \mathrm{~cm}^{-1}$ corresponded to the asymmetric and symmetric vibrational stretching of $\mathrm{C}=\mathrm{O}$. The peak at $1347 \mathrm{~cm}^{-1}$ was due to vibrational stretching of C-N. The peaks at 1099 and $1031 \mathrm{~cm}^{-1}$ corresponded to the asymmetric and symmetric vibrational stretching of the sulfonic acid group $(S=O)$. The peak height of Vas $(S=O)$ and Vs $(S=O)$ increased linearly with the SD of the SPI membranes, except for SPI30 (Figure 3b), confirming that the SD was approximately equal to the BDSA feed because the ATR-FTIR results can
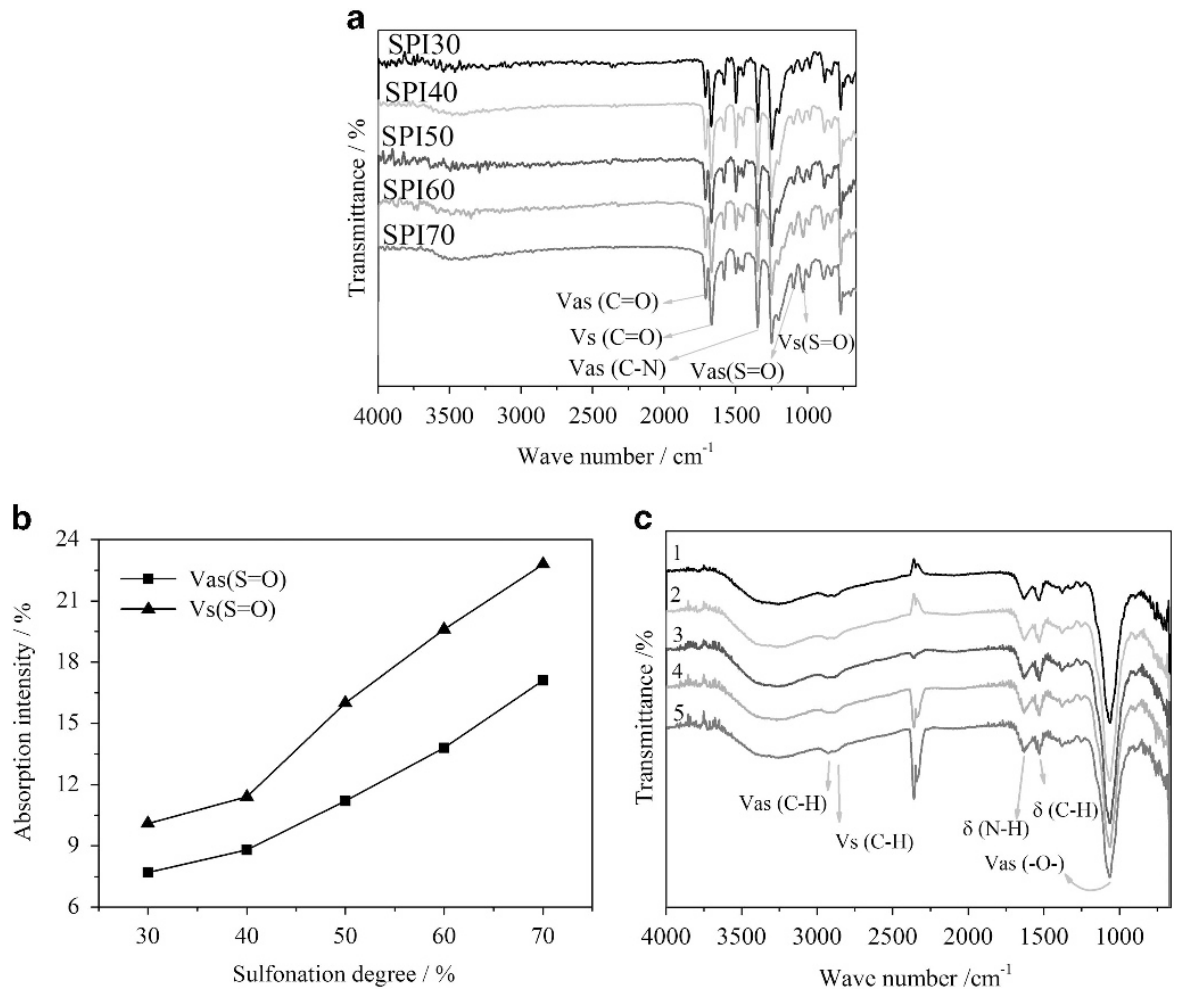

Figure 3 (a) Attenuated total reflection Fourier transformed infrared spectroscopy (ATR-FTIR) spectra of sulfonated polyimide (SPI) membranes with different sulfonation degrees. (b) Infrared absorption intensity of Vas $(S=0)$ (asymmetric vibration of $S=0$ ) and $V s(S=0)$ (symmetric vibration of $S=0$ ) for the $S P I$ membranes with different sulfonation degrees. (c) ATR-FTIR spectra of SPI/chitosan (CS) membranes with different sulfonation degrees (1, SPI30/CS; 2, SPI40/CS; 3, SPI50/CS; 4, SPI60/CS; 5, SPI70/CS). A full color version of this figure is available at Polymer Journal online. 
only semiquantitatively characterize the functional group content. The ATR-FTIR spectra of the SPI/CS membranes are shown in Figure 3c. The peaks at 2933 and $2884 \mathrm{~cm}^{-1}$ corresponded to the asymmetric and symmetric vibrational stretching of the methylene group (H-C-H) in CS. The peaks at 1626 and $1520 \mathrm{~cm}^{-1}$ were due to the bending vibrations of the amino $(\mathrm{N}-\mathrm{H})$ and methylene $(\mathrm{C}-\mathrm{H})$ groups of $\mathrm{CS}$, respectively. The peak at $1063 \mathrm{~cm}^{-1}$ was due to asymmetric vibrational stretching of the aliphatic ether bond (C-O-C) in the molecular chain of CS. This vibrational stretching peak of C-O-C in CS in the SPI/CS membrane is so wide and strong that it overlapped with the characteristic vibrational stretching of the sulfonic acid group $(S=O)$ in SPI. These ATR-FTIR spectra confirm the successful preparation of both the pristine SPI and SPI/CS composite membranes.

In addition, ${ }^{1} \mathrm{H}$ NMR spectroscopy (Figure 4) was also employed to investigate the chemical structure of the SPI membranes with different SDs. The chemical shift at 12.2 p.p.m. corresponding to the carboxyl peak was not observed for all of the SPI membranes that confirmed the complete imidization of SPI and is in agreement with the FTIR results. The chemical shifts at $7.43,7.77$ and 8.05 p.p.m. were due to BDSA, and the chemical shifts at 7.33 and 7.57 p.p.m. were due to ODA, as shown in Figure 4. The ratios of the integral area of the chemical shift at 8.05 p.p.m. to that at 7.57 p.p.m. were $27 \%, 39 \%$, $57 \%, 68 \%$ and $93 \%$ for SPI30, SPI40, SPI50, SPI60 and SPI70, respectively. Therefore, the calculated SDs for SPI30, SPI40, SPI50, SPI60 and SPI70 were $35.1 \%, 43.8 \%, 53.3 \%, 57.6 \%$ and $65.5 \%$, respectively, approximately similar to the designed SD. This result is also consistent with the results shown in Figure 3a that indicates the successful synthesis of SPI with different SDs.

\section{Morphological analysis}

Figures $5 \mathrm{a}-\mathrm{j}$ shows the surface images of the SPI30, SPI30/CS, SPI40, SPI40/CS, SPI50, SPI50/CS, SPI60, SPI60/CS, SPI70 and SPI70/CS membranes, respectively. Particles with a size of $\sim 50 \mathrm{~nm}$ were
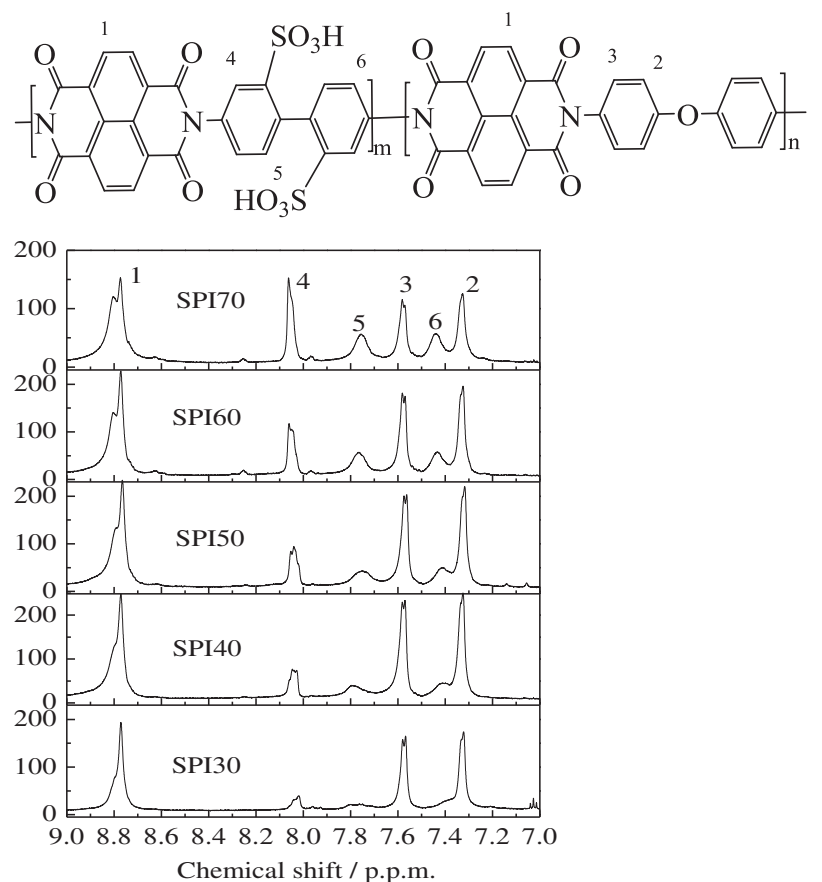

Figure $4{ }^{1} \mathrm{H}$ nuclear magnetic resonance $\left({ }^{1} \mathrm{H}\right.$ NMR) spectra of sulfonated polyimide (SPI) membranes with different sulfonation degrees. observed in the images of CS on the SPI, and these particles were equally distributed in the images of the SPI/CS composite membranes shown in Figures $5 \mathrm{~b}, \mathrm{~d}, \mathrm{f}, \mathrm{h}$ and $\mathrm{j}$. For the CS particles on the SPI membrane, studies of CS-based acid-base composite membranes by other groups ${ }^{15}$ do not report the observation of the existence of spherical CS particles on the composite membrane surface. Therefore, we cannot determine whether the formation of CS particles on SPI is common or not. However, Seyed et al. ${ }^{28}$ did observe similar spherical CS particles during the preparation of bioactive fish gel/CS nanoparticle composite films. The as-observed spherical CS particles on the SPI membrane may be caused by the CS itself as well as the preparation process of SPI/CS composite membrane. However, CS possesses a nanospherical morphology. In addition, the CS layer was deposited onto the SPI membrane using an infiltration and selfassembly method. During this preparation process, the CS particles were swollen in water and then dried by an oven that resulted in shrinkage and aggregation of CS particles. The pristine SPI membrane with a high SD exhibited a smoother surface based on a comparison of the SPI70 (RMS (root-mean-squared roughness) $=1.875 \mathrm{~nm}$ ) and SPI60 membranes $($ RMS $=2.855 \mathrm{~nm})$ to the SPI50 $($ RMS $=3.873 \mathrm{~nm})$, SPI40 $(\mathrm{RMS}=4.929 \mathrm{~nm})$ and SPI30 $(\mathrm{RMS}=5.324 \mathrm{~nm})$ membranes. In addition, the SPI30/CS and SPI40/CS composite membranes exhibited smoother surface compared with those of SPI30 and SPI40, indicating successful surface morphological modification of the acid-base interaction between SPI and CS. When the SD increased to 60 or $70 \%$, the surfaces of the SPI/CS composite membranes became rougher than those of the pristine SPI membranes because the SPI60 and SPI70 membranes have a very smooth surface. The addition of spherical CS particles onto the SPI membranes results in a slightly rougher surface for the SPI/CS composite membranes. However, all of the as-prepared SPI/CS composite membranes have suitably smooth surfaces that are applicable to VRFBs.

To gain additional insight into the cross-sectional morphology of the as-prepared composite membranes, scanning electron microscopy observations of the cross-sections of the SPI/CS membranes were performed, and the images are shown in Figures 6a-e, where the location of the CS layer is indicated by black parallel lines. The CS layers were closely combined onto the SPI membranes, and no cracks at the interface layer were observed for any of the SPI/CS membranes. The thickness of the CS layer on the SPI/CS membranes with various SDs (that is, 30, 40, 50, 60 and 70\%) was $\sim 1.5 \mu \mathrm{m}$. Therefore, the CS layer was successfully deposited onto the SPI membrane, and a sandwich-like structure was obtained in the SPI/CS membranes.

Ion exchange capacity, water uptake and swelling ratio The IEC indicates the $\mathrm{H}^{+}$exchange capacity of free $\mathrm{SO}_{3}{ }^{-}$in SPI. Because the content of CS in the SPI/CS composite membranes was very low, the effect of CS on the difference in IEC of the SPI/CS membranes is negligible, and the IEC values of the SPI/CS composite membranes are primarily dependent on the property of SPI. The measured IEC values that are shown in Figure 7 are in the following order: SPI30/CS (1.43 mequiv $g^{-1}$ dry) $<$ SPI40/CS (1.55 mequiv $g^{-1}$ dry) $<$ SPI50/CS (1.65 mequiv $g^{-1}$ dry) $<$ SPI60/CS (1.74 mequiv $g^{-1}$

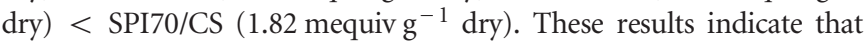
the IEC increased as the SD of the membranes increased ${ }^{7,9}$ because the content of sulfonic acid groups on the backbone of SPI increased gradually. The IEC of SPI70/CS was lower than the theoretically estimated value for SPI70 ( $>3$ mequiv $\mathrm{g}^{-1}$ dry) because the actual SD of SPI70 was lower than the theoretical value, as confirmed by ${ }^{1} \mathrm{H}$ NMR analysis (see Figure 4). In addition, the as-prepared SPI70/CS membrane is a type of acid-base composite membrane, and the 

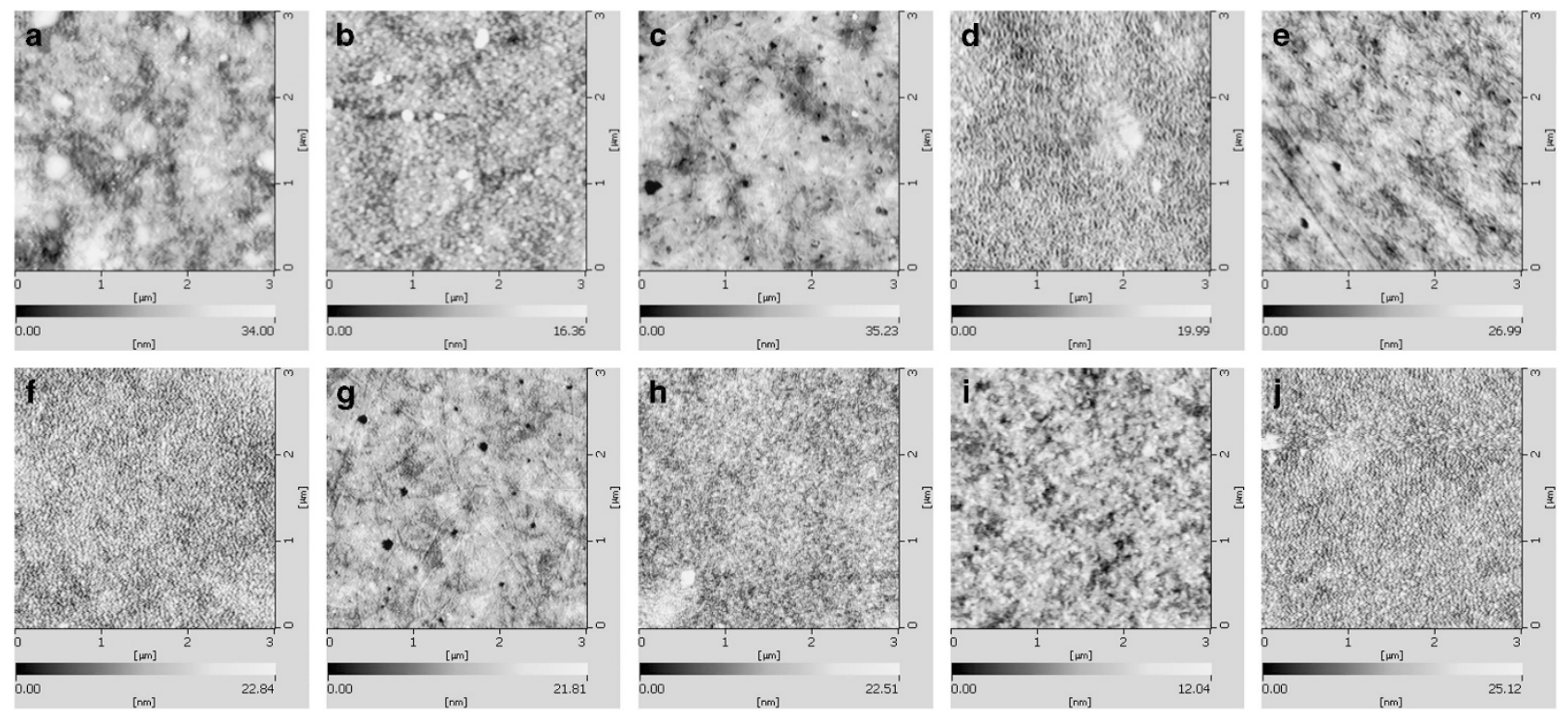

Figure 5 Atomic force microscope (AFM) phase images of sulfonated polyimide/chitosan (SPI/CS) membranes: (a) SPI30, (b) SPI30/CS, (c) SPI40, (d) SPI40/CS, (e) SPI50, (f) SPI50/CS, (g) SPI60, (h) SPI60/CS, (i) SPI70 and (j) SPI70/CS. A full color version of this figure is available at Polymer Journal online.

acid-base interaction decreases the content of free $-\mathrm{SO}_{3} \mathrm{H}$ groups, leading to a reduced IEC value for the SPI70/CS membrane. It is important to note that the IEC value of the SPI/CS membrane with a $\mathrm{SD}$ of zero (that is, SPI0/CS membrane) cannot be obtained via extrapolation of the line in the range of SDs from 30 to $70 \%$. However, this value could be obtained with a range of SD from 0 to $30 \%$. SPIs with a lower SD, such as SPI20, SPI10 and SPI5, are difficult to dissolve in $m$-cresol, and they were not successfully prepared as membranes. Therefore, the measured IEC values of the SPI20/CS, SPI10/CS and SPI5/CS membranes were not obtained. Therefore, the practical slope in the range of SDs from 0 to $30 \%$ for the SPI/CS membranes remains unknown. Nevertheless, the SPI30 membrane exhibited a higher practical SD than the theoretical value, as illustrated by the ${ }^{1} \mathrm{H}$ NMR spectrum (see Figure 4), and the trend is the opposite as that for the SPI70 membrane. Therefore, the slope of the fitted IEC line for the SPI/CS membranes in a SD range from 0 to $30 \%$ should be larger than that in a SD range from 30 to $70 \%$. Therefore, the extrapolation value of the $I E C$ at $0 \%$ sulfonation from the line in the range of SD from 0 to $30 \%$ for the SPI/CS membranes should be close to zero. For an extrapolated value from a SD range from 0 to $30 \%$ that is slightly larger than 0 , this difference may result from contributions of CS to the IEC of the SPI/CS membrane.

As shown in Figure 8, the water uptake of the SPI/CS membrane increased with the SD of SPI because water uptake is primarily dependent on the hydrophilicity of the membrane. In particular, for a membrane with more hydrophilic groups, the water uptake is higher. To explain why the swelling ratio is not proportional to the water uptake, the density of the dry SPI/CS membrane was measured and recorded in Table 3 . When the $\mathrm{SD}$ was $\geqslant 40 \%$, the relationship between $W U$ and $S W$ was $W U \approx\left(\rho_{\text {water }} / \rho_{\text {film }}\right) \times S W$. This result indicates that the SPI/CS membranes with high SDs are anisotropically swollen in the thickness direction because the SPI/CS membrane was confined to the in-plane direction by the hard CS. However, when the SD was as low as $30 \%$, the relationship between $W U$ and $S W$ was $W U$ $\approx 3\left(\rho_{\text {water }} / \rho_{\text {film }}\right) \times S W$, indicating that the SPI30/CS membrane was not anisotropically swollen but isotropically swollen. This exception may be due to the SPI30 membrane possessing a higher viscosity average molecular weight (see Table 2) that makes it harder than SPI membranes with higher SDs because of the low SD of SPI30. Therefore, the hard SPI30 composited with hard CS causes the SPI30/CS membrane to be isotropically swollen. In fact, this nonproportional relationship between the water uptake and the swelling ratio has been previously reported by Fengxiang et al. ${ }^{29}$ and Nanfang et al. ${ }^{30}$

\section{Proton conductivity and vanadium ion permeability}

Figure 9 shows the proton conductivity of different SPI/CS composite membranes, and the proton conductivity increased as the SD increased. In general, the proton conductivity is primarily determined by the IEC and also enhanced by an increase in the water content inside the membranes. In addition, more sulfonic acid groups on the SPI backbones result in a higher proton conductivity. Here, the SPI70/CS membrane exhibited the highest proton conductivity $\left(4.88 \times 10^{-2} \mathrm{~S} \mathrm{~cm}^{-1}\right)$, and this value is close to that of Nafion 117 $\left(5.82 \times 10^{-2} \mathrm{~S} \mathrm{~cm}^{-1}\right){ }^{8,10}$ The vanadium ion permeability of the SPI30/CS membrane was zero or too small to measure, and SPI70/CS exhibited the highest $\mathrm{VO}^{2+}$ permeability among all of the SPI/CS membranes. When the SD was $\geqslant 50 \%$, the vanadium ion permeability suddenly increased. As the SD increased, the water uptake, ion exchange capacity and swelling ratio of the SPI/CS membrane increased, as shown in Figures 7 and 8. In particular, the swelling ratio sharply increased for the SPI/CS membrane with a high $\mathrm{SD}$. More water molecules in the membrane increase the probability of vanadium ions getting close to and permeating into the membrane. More sulfonic acid groups in the membrane increase the quantity of positive ion exchange group, leading to higher vanadium ion permeation. Moreover, excessive swelling of the membrane may lead to an abrupt increase in the vanadium ion permeability. When tested with the same method, the Nafion 117 membrane exhibited a much higher $\mathrm{VO}^{2+}$ permeability $\left(17.10 \times 10^{-7} \mathrm{~cm}^{2} \mathrm{~min}^{-1}\right)^{8,10}$ compared with that of SPI70/CS $\left(10.47 \times 10^{-7} \mathrm{~cm}^{2} \mathrm{~min}^{-1}\right)$. These results 

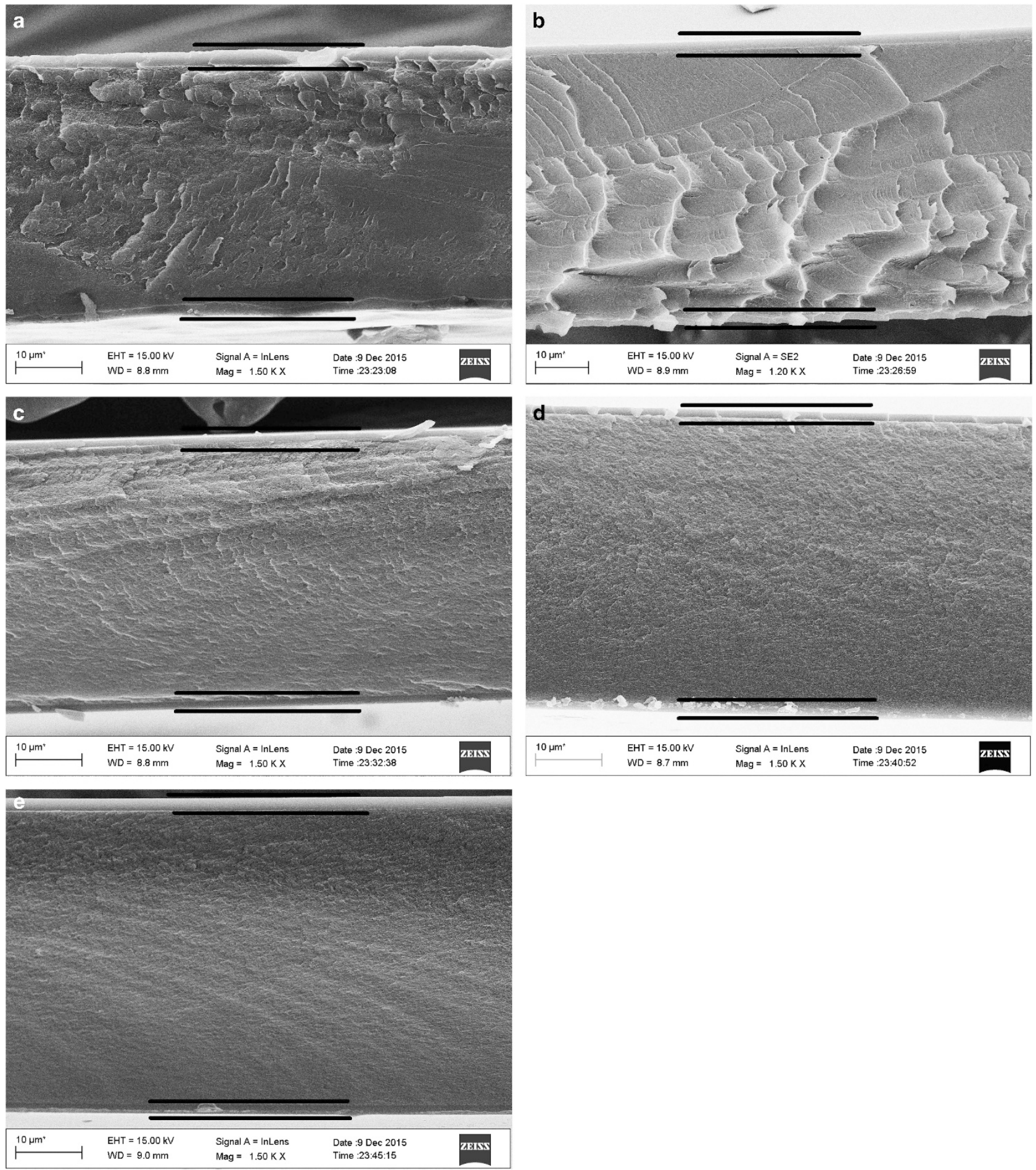

Figure 6 Cross-section images of sulfonated polyimide/chitosan (SPI/CS) membranes: (a) SPI30/CS, (b) SPI40/CS, (c) SPI50/CS, (d) SPI60/CS and (e) SPI70/CS

indicate that the as-prepared SPI/CS membranes exceed Nafion 117 with regard to vanadium ion resistance.

\section{Chemical stability and mechanical property}

Because no unified testing method for the chemical stability of VRFB membranes previously existed, ${ }^{1}$ the chemical stabilities of the SPI/CS membranes were investigated in an acidic $\mathrm{VO}_{2}{ }^{+}$solution at $40^{\circ} \mathrm{C}$.
As presented in our previous study, a similar chemical stability test was performed, ${ }^{17}$ and the results indicate that the SPI/CS membrane exhibited a lower weight loss than that of the SPI membrane in the $\mathrm{VO}_{2}{ }^{+}$solution, demonstrating that the chemical stability of the SPI/CS composite membrane is better than that of the pristine SPI membrane. Figure 10 shows digital photos of the SPI/CS composite membranes after immersion in $0.1 \mathrm{moll}^{-1} \mathrm{VO}_{2}^{+}$and $3.0 \mathrm{moll}^{-1} \mathrm{H}_{2} \mathrm{SO}_{4}$ mixed 


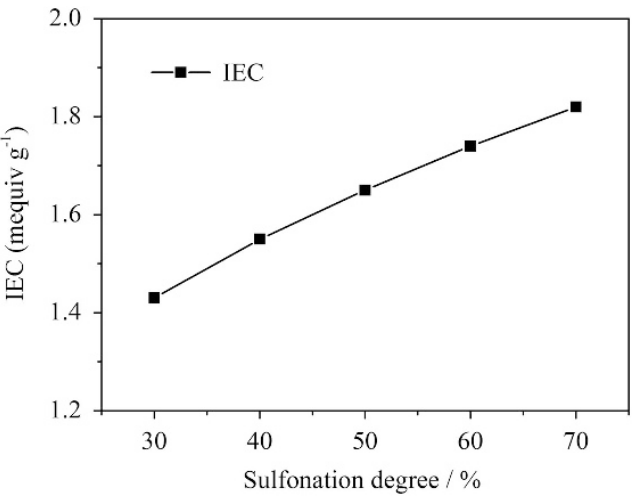

Figure 7 The ion exchange capacity (IEC) of sulfonated polyimide/chitosan (SPI/CS) membranes with different sulfonation degrees.

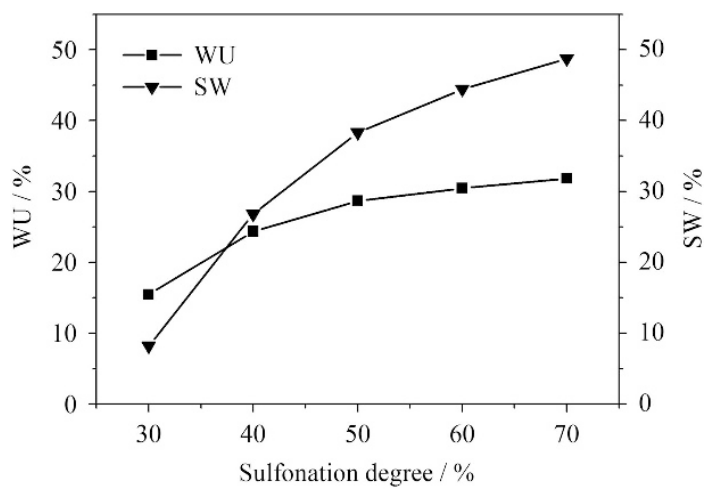

Figure 8 WU (water uptake) and SW (swelling ratio) of sulfonated polyimide/ chitosan (SPI/CS) membranes with different sulfonation degrees.

Table 3 Physicochemical properties of the SPI/CS and Nafion 117 membranes

\begin{tabular}{lccccc}
\hline Membrane & $\begin{array}{c}\text { Sulfonation } \\
\text { degree }\end{array}$ & $\begin{array}{c}\text { CS content } \\
(\%)\end{array}$ & $\begin{array}{c}\text { Thickness } \\
(\mu \mathrm{m})\end{array}$ & $\begin{array}{c}\text { Density } \\
\left(\mathrm{g} \mathrm{cm}^{-3}\right)\end{array}$ & $\begin{array}{c}\text { Duration } \\
\text { time (min) }\end{array}$ \\
\hline SPI30/CS & $30 \%$ & 8.54 & 50 & 1.34 & 1210 \\
SPI40/CS & $40 \%$ & 5.65 & 48 & 1.41 & 970 \\
SPI50/CS & $50 \%$ & 5.74 & 50 & 1.49 & 790 \\
SPI60/CS & $60 \%$ & 7.55 & 50 & 1.59 & 660 \\
SPI70/CS & $70 \%$ & 7.04 & 50 & 1.68 & 600 \\
Nafion 117 & - & - & 175 & - & - \\
\hline
\end{tabular}

Abbreviations: CS, chitosan; Duration time, the endurance time when membranes begin to break in Fenton's reagent; SPI, sulfonated polyimide.

aqueous solution (a little dark yellow) at $40{ }^{\circ} \mathrm{C}$ for $0,5,10$ and 15 days. All of the membranes appear to be intact in these solutions. A few $\mathrm{VO}^{2+}$ ions were produced because of the reaction between the SPI/CS membrane and the $\mathrm{V}(\mathrm{V})$ ions in the acidic $\mathrm{V}(\mathrm{V})$ solution, causing a gradual change in the color of the solution. However, after the membranes were removed from colorimetric tubes after 15 days, the SPI60/CS and SPI70/CS membranes were very fragile and even broke into pieces, as shown in Figure 11. However, the mechanical properties of SPI30/CS and SPI40/CS were much better. ${ }^{31}$ Then, the chemical stability was characterized in Fenton's reagent, and the results are shown in Table 3. The time that the SPI/CS membrane endures the Fenton's reagent increased as the SD of SPI decreased, indicating that a higher SD results in poorer chemical stability. The results from both chemical stability testing methods indicate that the SPI/CS membrane

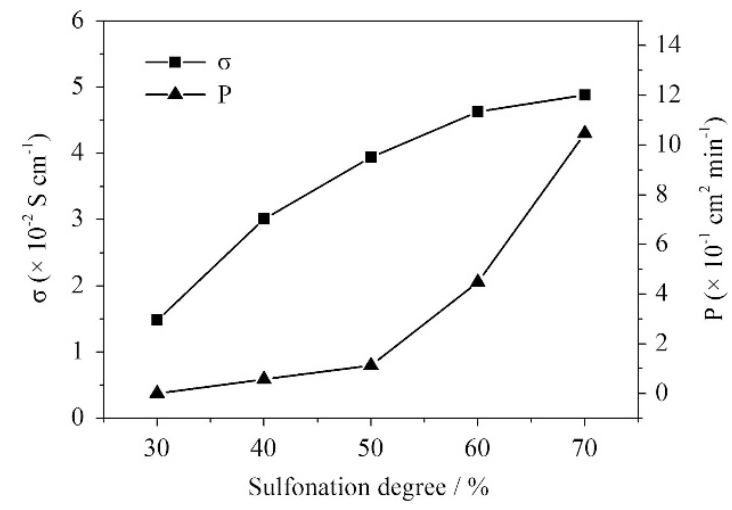

Figure 9 The $\sigma$ (proton conductivity) and $P\left(\mathrm{VO}^{2+}\right.$ permeability coefficient) of sulfonated polyimide/chitosan (SPI/CS) membranes with different sulfonation degrees.

with a low SD exhibits better chemical stability. This result is because of the water uptake of the SPI/CS membrane with the low SD being lower than that of the SPI/CS membrane with the high SD, leading to fewer opportunities for attack by $\mathrm{VO}_{2}{ }^{+}$and hydroxyl radicals. ${ }^{25,32}$

We are very interested in the decomposition mechanism of the SPI/CS membrane in the offline chemical stability tests. Therefore, we carried out FTIR analysis of the SPI/CS membrane before and after decomposition in both the acidic vanadium ion solution and Fenton's reagent. However, the peak corresponding to - $\mathrm{COO}\left(1780 \mathrm{~cm}^{-1}\right.$ or so theoretically) was not observed, as shown in Figure 12, because the quantity of carboxylic acid group may have been too low. Therefore, the ${ }^{1} \mathrm{H}$ NMR analysis was used to clarify the possible degradation mechanism.

The crosslinked CS was difficult to solve in the dimethyl sulfoxide solvent during the ${ }^{1} \mathrm{H}$ NMR measurement. To clarify whether the degradation of SPI was due to the hydrolysis of the imide linkage or oxidative decomposition, the oxidative stability of the pristine SPI50 membrane as a representative was investigated using two methods. First, the SPI50 membrane was immersed into an acidic $\mathrm{VO}_{2}{ }^{+}$ solution at $40^{\circ} \mathrm{C}$ for $360 \mathrm{~h}$, and then the SPI50 membrane was immersed into Fenton's reagent at $80^{\circ} \mathrm{C}$ for $24 \mathrm{~h}$. The structures of the degraded SPI50 membranes after immersion in the acidic $\mathrm{VO}_{2}{ }^{+}$ solution and Fenton's reagent were confirmed by ${ }^{1} \mathrm{H}$ NMR spectroscopy (Figure 13). In comparison to the initial membrane, the degraded SPI50 membranes that were immersed in the acidic $\mathrm{VO}_{2}{ }^{+}$ solution and Fenton's reagent exhibited a new peak with a chemical shift at 12.16 p.p.m. (carboxylic acid), indicating that the $-\mathrm{COOH}$ group is a degradation product that is produced during both chemical stability tests.

Based on the presence of $-\mathrm{COOH}$ as a degradation product, the decomposition was most likely caused by hydrolysis of SPI. Gills et al. ${ }^{27}$ reported that the SPI membrane for fuel cell applications is primarily degraded by hydrolysis, and this degradation process can be accelerated by hydroxyl radicals, but the effect of hydroxyl radicals on the hydrolysis process of SPI remains unknown. Yuan et al..$^{32}$ also observed $-\mathrm{COOH}$ groups in degradation products when studying the degradation mechanism of a polyoxadiazole membrane in an acidic $\mathrm{VO}_{2}{ }^{+}$solution. The Yuan group confirmed that the existence of oxygen species participated in opening of the oxadiazole ring when $\mathrm{V}(\mathrm{V})$ was reduced to $\mathrm{V}(\mathrm{IV})$. In addition, a significant change in the color of the acidic vanadium solution was observed because of the change in the valence of the vanadium ions. Therefore, Yuan et al. ${ }^{32}$ concluded that the polyoxadiazole membrane was oxidatively 

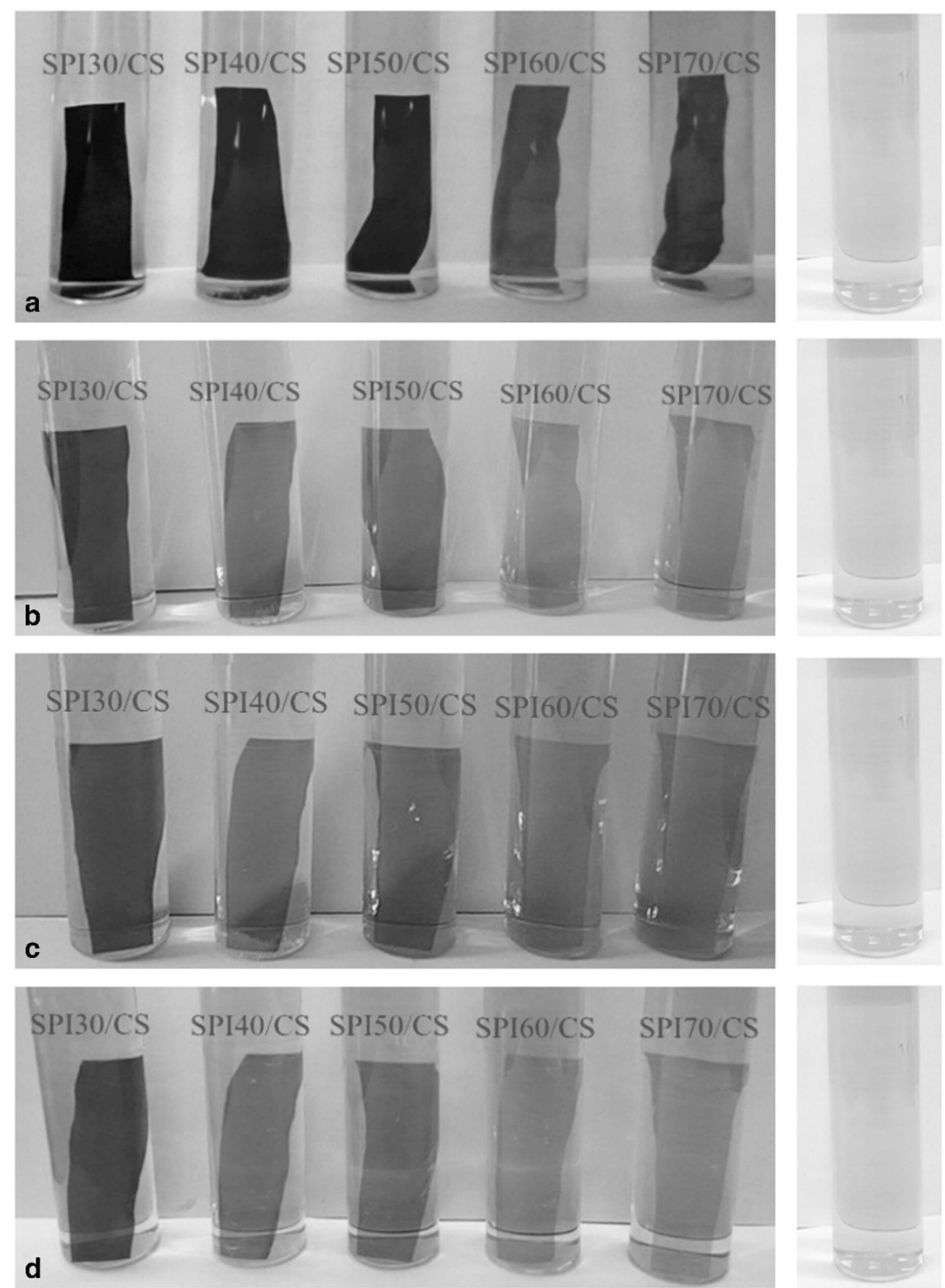

Figure 10 Digital photos of sulfonated polyimide/chitosan (SPI/CS) membranes immersed in $0.1 \mathrm{~mol} \mathrm{I}^{-1} \mathrm{VO}_{2}{ }^{+}+3 \mathrm{~mol} \mathrm{I}^{-1} \mathrm{H}_{2} \mathrm{SO}_{4}$ solutions at $40{ }^{\circ} \mathrm{C}$ for (a) 0 day, (b) 5 days, (c) 10 days and (d) 15 days. A full color version of this figure is available at Polymer Journal online.
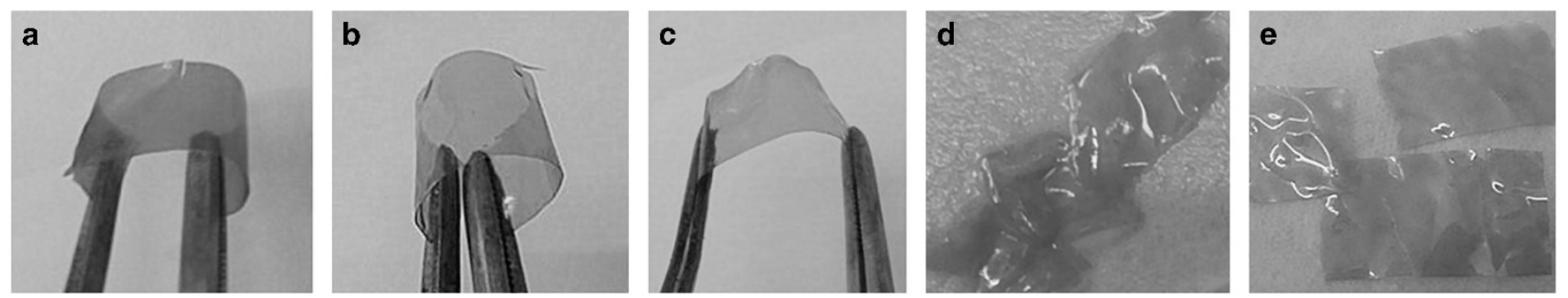

Figure 11 Digital photos of sulfonated polyimide/chitosan (SPI/CS) membranes after removal from the $0.1 \mathrm{moll}^{-1} \mathrm{VO}_{2}{ }^{+}+3 \mathrm{~mol}^{-1} \mathrm{H}_{2} \mathrm{SO}_{4} \mathrm{solutions}$ at $40{ }^{\circ} \mathrm{C}$ after 15 days. (a) SPI30/CS, (b) SPI40/CS, (c) SPI50/CS, (d) SPI60/CS and (e) SPI70/CS. A full color version of this figure is available at Polymer Journal online.

degraded in the acidic vanadium ion solution. In fact, the exact degradation mechanism of the SPI/CS membranes in the acidic vanadium ion solution is difficult to clarify because of the complexity of this type of composite membrane, and few studies of the degradation mechanism of acid-base composite membranes for VRFB applications have been reported. Based on the ${ }^{1} \mathrm{H}$ NMR analysis and the color change of the acidic vanadium ion solution along with the detrimental morphological destruction of the surface facing the 


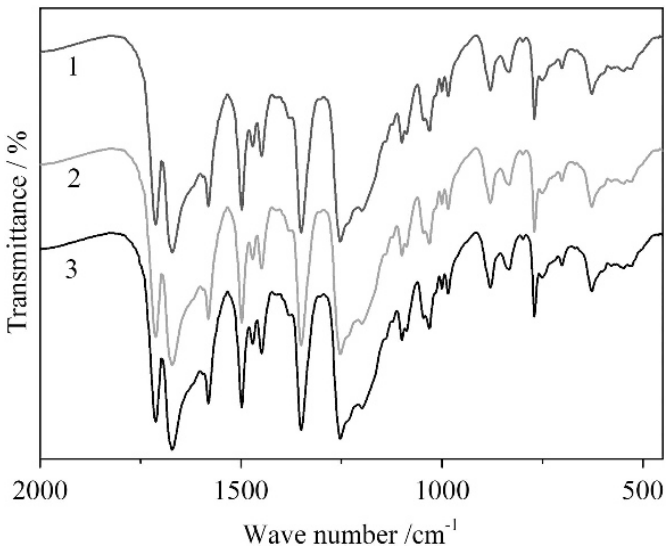

Figure 12 Fourier transformed infrared spectroscopy (FTIR) spectra of: 1, initial SPI50/CS membrane; 2, SPI50/CS membrane treated with Fenton's reagent at $80^{\circ} \mathrm{C}$ for $24 \mathrm{~h}$; and 3 , SPI50/CS membrane treated with acidic vanadium ion solution at $40^{\circ} \mathrm{C}$ for 15 days. SPI/CS, sulfonated polyimide/chitosan. A full color version of this figure is available at Polymer Journal online.

positive electrolyte (acidic $\mathrm{V}(\mathrm{V})+\mathrm{V}(\mathrm{IV})$ solution) compared with that facing the negative electrolyte (acidic V(II) $+\mathrm{V}(\mathrm{III})$ solution), SPI is most likely degraded by oxidation and/or hydrolysis. Because this study is more focused on the effect of the SD of SPI on the properties of the SPI/CS composite membranes, further studies on the decomposition mechanism of SPI/CS membranes in the offline chemical stability measurement will be conducted in the near future. In addition, both chemical stability results are offline, and the chemical stability of the SPI/CS membrane in real VRFBs is important. Therefore, the decomposition mechanism of the SPI/CS membrane under actual VRFB conditions is currently under way in our laboratory.

The mechanical properties of the SPI/CS and SPI membranes with different SDs were characterized by their maximum stress at break $(S)$, and the data are shown in Figure 14. The maximum stress at break for the SPI/CS membranes with different SDs were higher than that of pure SPI, demonstrating the improved mechanical strength that resulted from compositing a CS layer onto a SPI membrane. However, the maximum stress at break decreased as the SD of the SPI/CS or SPI membranes increased because the water swelling ratio increased as the SD increased, leading to a decrease in the intermolecular interactions.

\section{Single VRFB performance}

Because the SPI30/CS membrane exhibited negligible $\mathrm{VO}^{2+}$ permeability, as shown in Figure 9, we investigated its VRFB performance, and the charge-discharge testing for the SPI30/CS membrane was conducted. The results indicate that the VRFB with the SPI30/CS membrane cannot be charged normally at high current densities, such as 20,30 and $40 \mathrm{~mA} \mathrm{~cm}^{-2}$, under the current experimental conditions because of its proton conductivity being too low. Therefore, the charge-discharge testing was only carried out at $10 \mathrm{~mA} \mathrm{~cm}^{-2}$, and the battery efficiency as well as the charge capacity retention results are shown in Figures 15a and b. Because the current density was very low, the charge-discharge process was very slow, and $360 \mathrm{~h}$ were required to complete 30 cyclic charge-discharge tests for the SPI30/CS membrane. During this long testing period, no decline in $C E, V E$ and $E E$ was observed (Figure 15a), indicating that the SPI30/CS membrane possesses good cycling battery efficiency and excellent chemical stability to survive the severe testing environment. However,
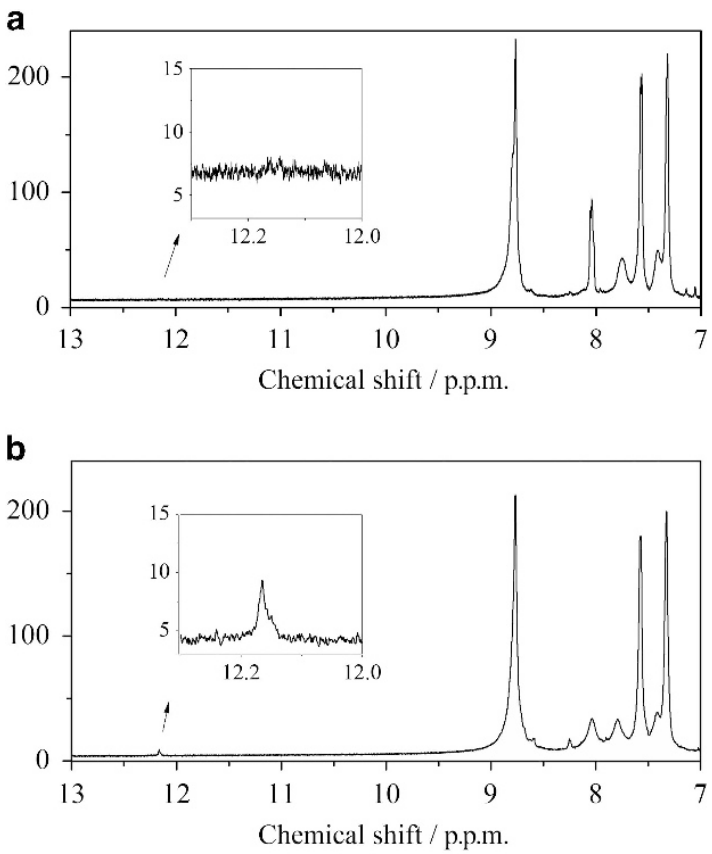

c

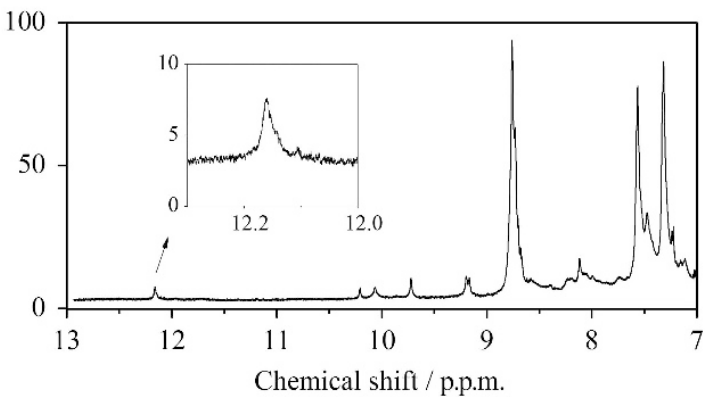

Figure $13{ }^{1} \mathrm{H}$ nuclear magnetic resonance $\left({ }^{1} \mathrm{H}\right.$ NMR) spectra of (a) initial SPI50 membrane, (b) degraded SPI50 membrane immersed in $0.1 \mathrm{M} \mathrm{V}^{5+}+$ $3 \mathrm{M} \mathrm{H}_{2} \mathrm{SO}_{4}$ aqueous solution and (c) degraded SPI50 membrane immersed in Fenton's reagent. SPI/sulfonated polyimide.

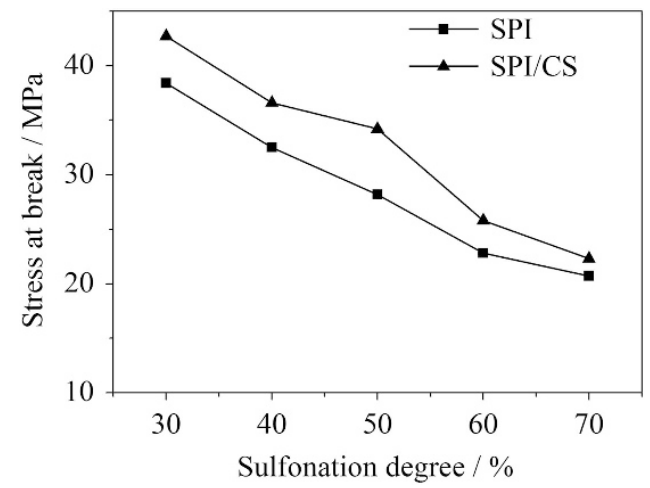

Figure 14 Stress at break (S) of sulfonated polyimide (SPI) and SPI/chitosan (CS) membranes with different sulfonation degrees in the wet state.

the charge capacity retention was $<18.2 \%$ after 30 cycles, as shown in Figure $15 \mathrm{~b}$. The capacity decay was primarily because of the undesired transport of vanadium ions across the membrane during the charge-discharge operation. ${ }^{14,33}$ As previously mentioned, the $\mathrm{VO}^{2+}$ permeability of SPI30/CS was close to zero in the offline vanadium ion 
permeability measurement but this result appears to conflict with the online VRFB testing results. This conflict is primarily because of the difference in the testing condition between the offline vanadium ion permeability and online cyclic charge-discharge experiments. The concentration difference is the main driving force for vanadium ion permeability during the offline measurement but the electric potential is added as an additional driving force during the online VRFB testing. The driving force in the online test was larger than that in the offline
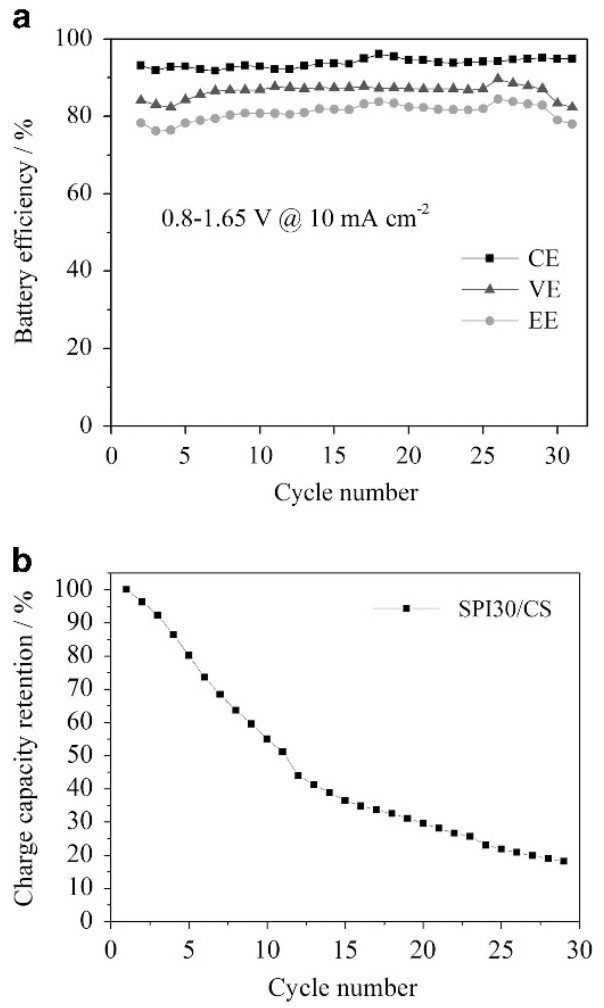

Figure 15 Cycling performance of the vanadium redox flow battery (VRFB) with the SPI30/CS membrane. (a) Efficiency and (b) charge capacity retention. SPI/CS, sulfonated polyimide/chitosan. A full color version of this figure is available at Polymer Journal online. test. Therefore, the vanadium ion permeability increased in the online VRFB measurements. Besides, the vanadium ions are only $\mathrm{VO}^{2+}$ in the offline vanadium ion permeability test. Nevertheless, the permeated vanadium ions may be $\mathrm{VO}^{2+}, \mathrm{VO}_{2}{ }^{+}, \mathrm{V}^{2+}$ and $\mathrm{V}^{3+}$ in the online test. Vanadium ions with different valances may exhibit different permeabilities across the SPI30/CS membrane, ${ }^{34}$ and this would also lead to an increase in the vanadium ion permeability in the online test. To further explain the vanadium ion permeability difference between the offline permeability and online VRFB tests, additional studies are urgently needed.

In addition, another interesting experimental phenomenon was observed during the VRFB tests for the SPI30/CS membrane. The color of the positive electrolyte changes from dark (a combined color consisting of blue $\left(\mathrm{VO}^{2+}\right)$ and yellow $\left(\mathrm{VO}_{2}^{+}\right)$) to bright yellow in each cycling charging process, as shown in Figure 16, indicating that an overwhelming majority of the vanadium ions in the positive electrolyte are $\mathrm{VO}_{2}{ }^{+}$at the end of the charging process. For example, the $\mathrm{VO}^{2+}$ to $\mathrm{VO}_{2}{ }^{+}$ratio at the end of the second charging process of the cycle test for the SPI30/CS membrane was measured to be $0.64 \%$. Then, the same cycle test of the VRFB with the SPI40/CS and SPI60/CS membranes was carried out at $10 \mathrm{~mA} \mathrm{~cm}{ }^{-2}$, and the $\mathrm{VO}^{2+}$ to $\mathrm{VO}_{2}{ }^{+}$ ratio in the positive electrolyte at the end of the second charging process was as high as $5.16 \%$ and $13.73 \%$, respectively. When the $\mathrm{V}^{4+}$ to $\mathrm{V}^{5+}$ ratio in the positive electrolyte was smaller, the color of the positive solution was more yellow. Therefore, the color of the positive electrolyte changes from a dark color to a bright yellow color during the charging process for each charge-discharge cycle was only observed for the SPI30/CS membrane, and the color of the positive electrolyte was a little blue green at the end of the charging process for the other SPI/CS membranes. This phenomenon has not been previously reported for the VRFB membranes with low vanadium ion permeability. This result motivated us to further understand the mechanism of the capacity decay during the cyclic charge-discharge tests for these types of membranes, ${ }^{5,35}$-39 and this study is also currently under way in our laboratory.

The performances of single VRFBs with SPI40/CS, SPI50/CS, SPI60/CS and SPI70/CS at different charge-discharge current densities were also investigated. Their charge-discharge curves are shown in Figures $17 \mathrm{a}-\mathrm{d}$. A high current density leads to strong polarization as well as a low charge and discharge capacity. ${ }^{12}$ As typically observed in

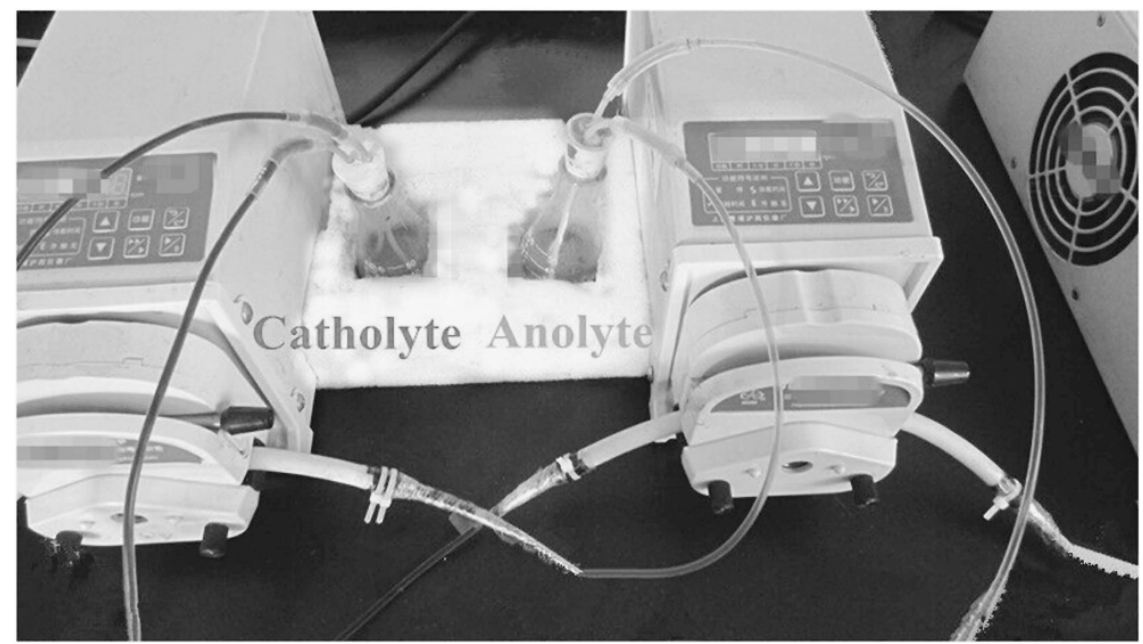

Figure 16 Images of the electrolytes in the vanadium redox flow battery (VRFB) with the SPI30/CS membrane at the end of the 30th charging process. $\mathrm{SPI} / \mathrm{CS}$, sulfonated polyimide/chitosan. A full color version of this figure is available at Polymer Journal online. 

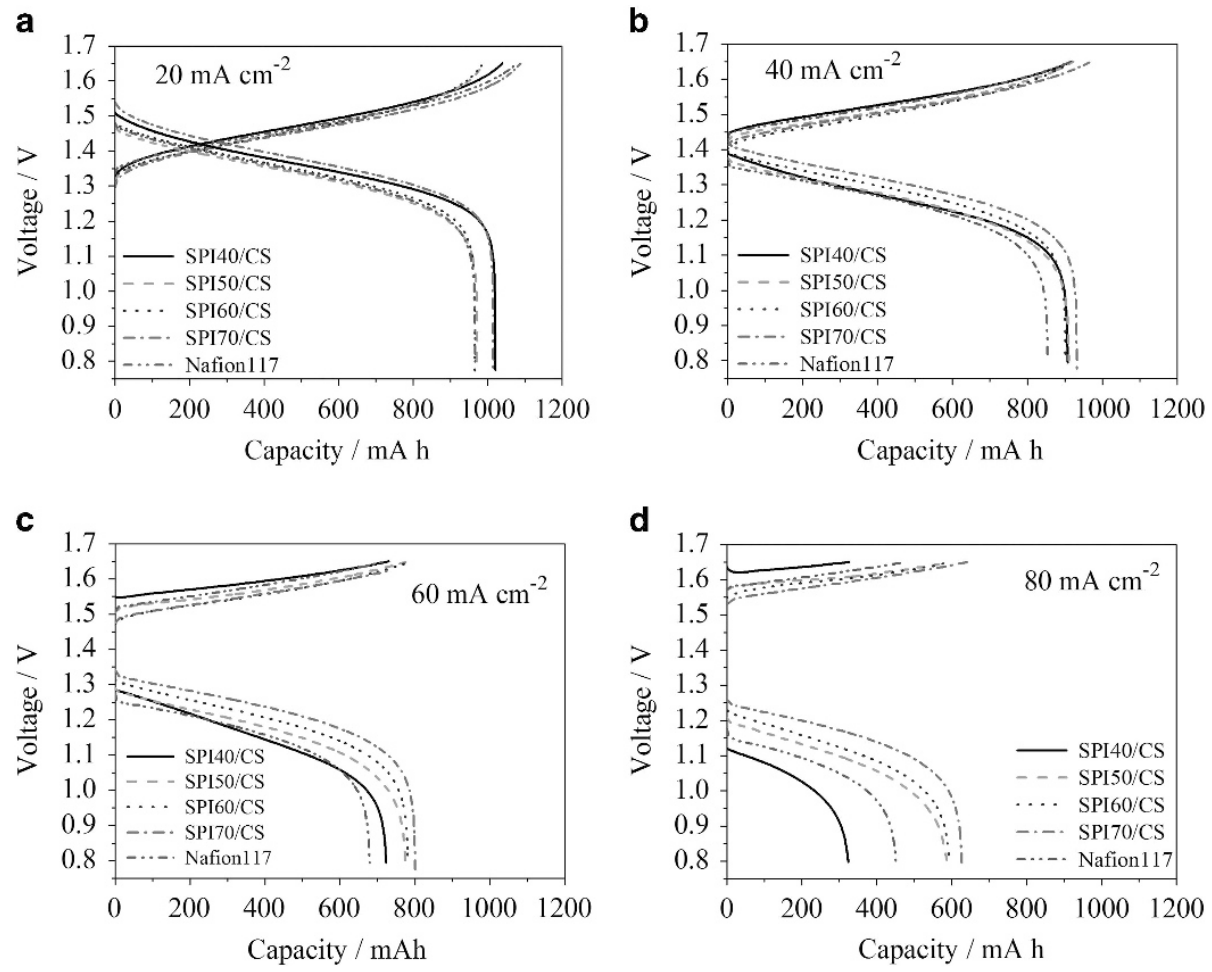

Figure 17 Charge-discharge curves of vanadium redox flow batteries (VRFBs) with the sulfonated polyimide/chitosan (SPI/CS) and Nafion 117 membranes at a current density of (a) $20 \mathrm{~mA} \mathrm{~cm}^{-2}$, (b) $40 \mathrm{~mA} \mathrm{~cm}^{-2}$, (c) $60 \mathrm{~mA} \mathrm{~cm}^{-2}$ and (d) $80 \mathrm{~mA} \mathrm{~cm}^{-2}$. A full color version of this figure is available at Polymer Journal online.
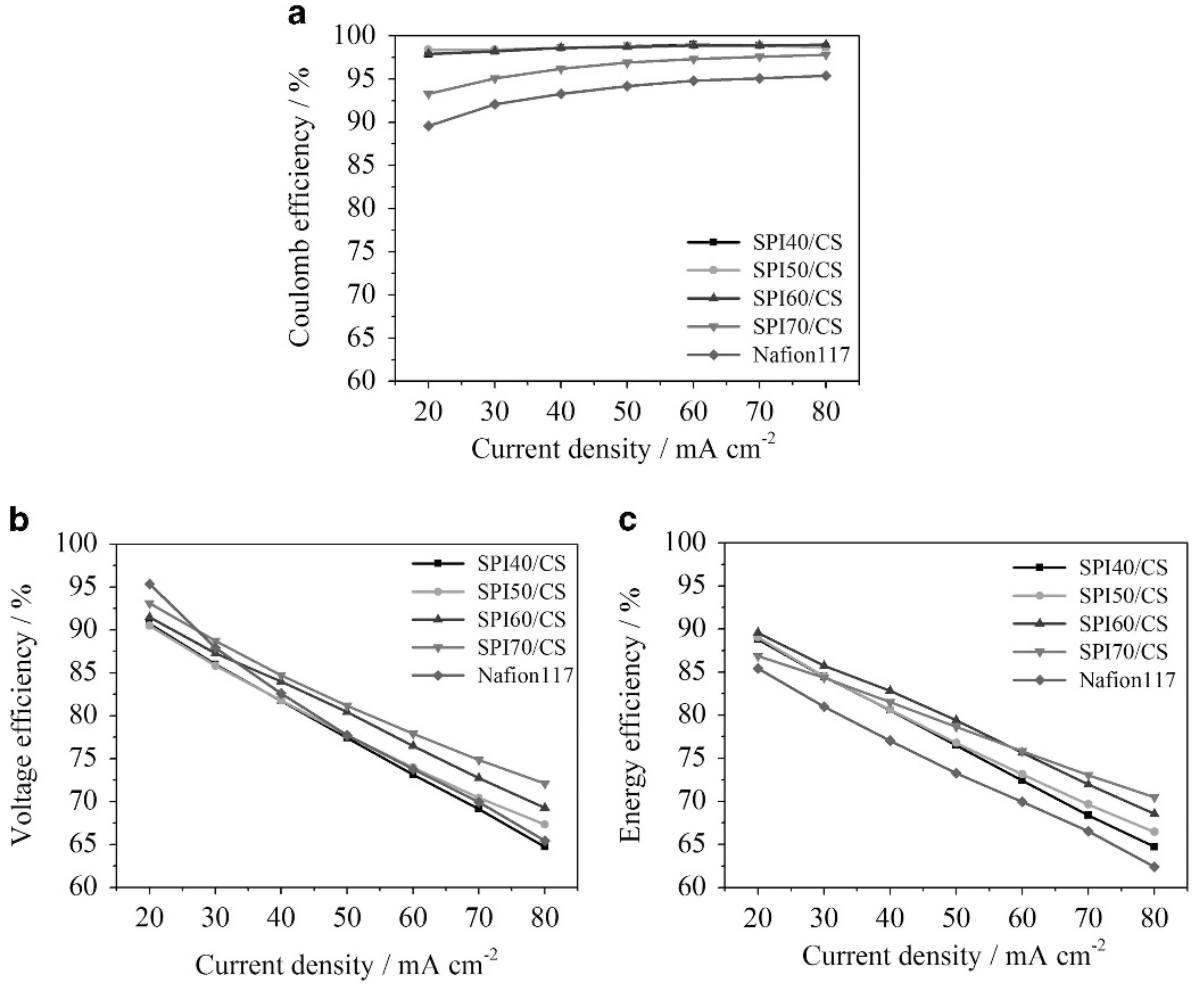

Figure 18 Battery efficiency of vanadium redox flow batteries (VRFBs) assembled with sulfonated polyimide/chitosan (SPI/CS) and Nafion 117 membranes at different current densities. (a) Coulombic efficiency (CE), (b) voltage efficiency (VE) and (c) energy efficiency (EE). A full color version of this figure is available at Polymer Journal online. 

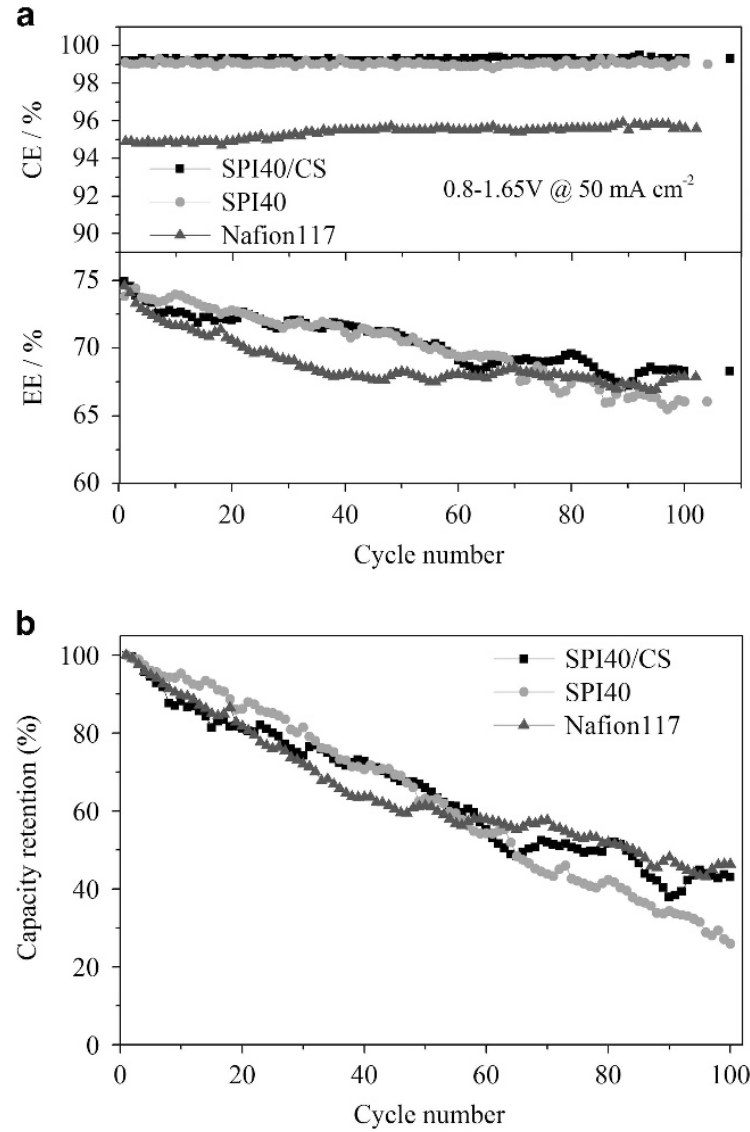

Figure 19 Cycling performance of vanadium redox flow batteries (VRFBs) with SPI40, SPI40/CS and Nafion 117 membranes. (a) Efficiency and (b) charge capacity retention. SPI/CS, sulfonated polyimide/chitosan. A full color version of this figure is available at Polymer Journal online.

other studies, the differences between the charge and discharge voltages typically increased at higher current densities because of ohmic polarization..$^{9,11,40}$ The battery efficiency results are shown in Figures 18a-c. The CE of SPI40/CS (98.3-99\%), SPI50/CS (98.4-98.7\%), and SPI60/CS (97.9-99\%) were similar to and higher than those of SPI70/CS and Nafion 117 at seven charge-discharge current densities (Figure 10a), in agreement with the offline vanadium ion permeability results shown in Table 3 and similar to previously reported results. ${ }^{41}$ Because the CE for the SPI40/CS, SPI50/CS and SPI60/CS membranes are as high as $\sim \geqslant 98 \%$, only slight variation in $C E$ as a function of the current density was observed. Only the $C E$ of SPI70/CS and Nafion 117 decreased from $97.8 \%$ and $95.4 \%$, respectively, to $93.3 \%$ and $89.6 \%$, respectively, when the current density varied from 80 to $20 \mathrm{~mA} \mathrm{~cm}{ }^{-2}$. This decrease may be because of the higher vanadium ion permeability, resulting from the higher charge-discharge time at lower current density. ${ }^{9}$ The $V E$ of the four SPI/CS membranes decreased as the current density increased because of an increase in the ohmic polarization of the membrane, as shown in Figure 18b. In general, the SPI/CS membrane with a higher SD has a higher $V E$ because of its higher proton conductivity. The EE of the SPI40/CS, SPI50/CS, SPI60/CS and SPI70/CS membranes at different current densities increased with the SD, especially at high current densities, as shown in Figure 18c. The $E E$ is the product of $C E$ and $V E$, as shown in Equation (9). The $E E$ is high or low depending on which efficiency dominates. The difference in EE among these SPI/CS membranes is primarily dependent on the ohmic resistance variation

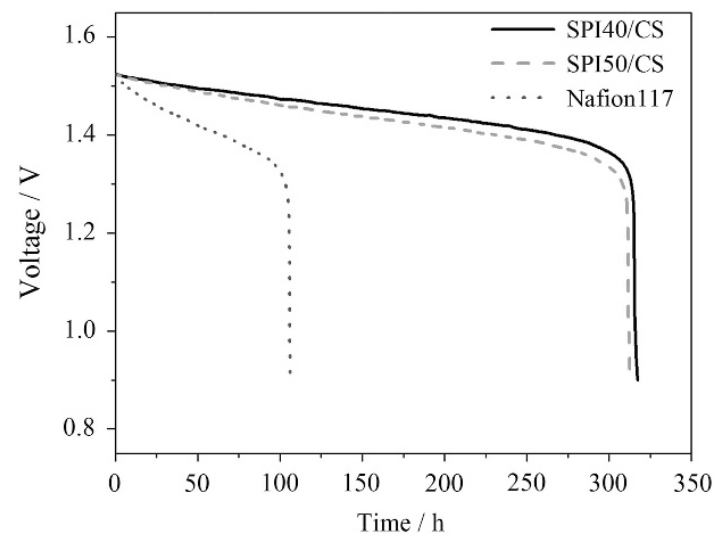

Figure 20 Open circuit voltages of the vanadium redox flow batteries (VRFBs) assembled with SPI40/CS, SPI50/CS and Nafion 117 membranes. $\mathrm{SPI}$ CS, sulfonated polyimide/chitosan. A full color version of this figure is available at Polymer Journal online.

in this study. Therefore, all of the as-prepared SPI/CS membranes exhibited higher EEs than that of Nafion 117 because the Nafion 117 membrane suffers from a much higher vanadium ion permeability compared with those of the SPI/CS membranes. These experimental $E E$ results indicate the potential of the as-prepared SPI/CS membranes for application in VRFBs.

In addition, the charge-discharge tests with the SPI40, SPI40/CS and Nafion 117 membranes were cycled 100 times at $50 \mathrm{~mA} \mathrm{~cm}^{-2}$. The battery efficiency and charge capacity retention changed with the cycle time, as shown in Figures 19a and b. The results indicate that the average $C E$ value for 100 cycles for the SPI40/CS membrane (99.3\%) is similar to that for SPI40 (99.0\%), and the average EE value for 100 cycles for the SPI40/CS membrane (70.5\%) is also similar to those for SPI40 (70.1\%) and Nafion 117 (68.86\%). For the capacity retention shown in Figure 19b, the SPI40/CS membrane remains very similar to the trend for Nafion 117. However, the SPI40 membrane exhibited a faster capacity decay than those of the Nafion 117 and SPI40/CS membranes, especially after $\sim 60$ charge-discharge cycles. In addition, in our previous study ${ }^{17}$ the battery efficiencies of the SPI50/CS and SPI50 membranes were also tested at a current density of $40 \mathrm{~mA} \mathrm{~cm}^{-2}$. The results indicate that the CE for the SPI50/CS membrane $(97.8 \%)$ is slightly higher than that for SPI50 (94\%), and the EE for the SPI50/CS membrane (88.6\%) is also slightly higher than that for SPI50 (86.7\%). Therefore, based on the results in this study and our earlier work, the as-prepared SPI/CS composite membrane exhibited better battery performance than that of the pure SPI membrane.

Under actual VRFB operational conditions, the open circuit voltage can also be used to imply the crossover rates of vanadium ions at continuous declining states of charges. As shown in Figure 20, the state of self-discharge is related to the vanadium ion permeability. Based on the slopes of the voltage decline and duration of self-discharge, the VRFBs assembled with SPI40/CS and SPI50/CS suffer less from self-discharge than that with Nafion 117.

\section{CONCLUSIONS}

A series of SPI/CS composite membranes with SDs ranging from 30 to $70 \%$ were successfully prepared, and their VRFB performances were also evaluated. The results indicate that the VRFB using SPI70/CS has the lowest $C E$ at all of the measured current densities as well as the highest EE at a high current density. However, the SPI70/CS membrane exhibited the worst chemical stability. The SPI60/CS 
membrane also exhibited a satisfactory $E E$ but an unacceptable chemical stability. The SPI30/CS, SPI40/CS and SPI50/CS membranes exhibited satisfactory $C E, E E$ and chemical stability. However, the SPI30/CS membrane possessed a proton conductivity that was too low for use in practical VRFBs. These results indicate that SPI with excessively high SDs leads to low durability of the SPI/CS composite membranes in VRFBs, and excessively low SDs lead to low energy and voltage efficiency in VRFBs. Therefore, based on the VRFB performance and the chemical stability, both the SPI40/CS and SPI50/ CS membranes have the potential for use as alternatives to the Nafion 117 membrane for VRFB applications.

\section{CONFLICT OF INTEREST}

The authors declare no conflict of interest.

\section{ACKNOWLEDGEMENTS}

Financial support from the National Natural Scientific Foundation of China (no. 21206138), the Key Fund Project of Sichuan Provincial Department of Education (no. 12ZA181), the Application Foundation Project of Science and Technology of Department of Sichuan Province (no. 2016JY0220), and the Professional Research and Innovation Team Construction Fund of Southwest University of Science and Technology (no. 14tdsc02) are greatly appreciated.

1 Li, X., Zhang, H., Mai, Z., Zhang, H. \& Vankelecom, I. Ion exchange membranes for vanadium redox flow battery (VRB) applications. Energ. Environ. Sci. 4, 1147 (2011).

2 Cunha, Á., Martins, J., Rodrigues, N. \& Brito, F. P. Vanadium redox flow batteries: a technology review. Int. J. Energ. Res. 39, 889-918 (2015).

3 Kear, G., Shah, A. A. \& Walsh, F. C. Development of the all-vanadium redox flow battery for energy storage: a review of technological, financial and policy aspects. Int. J. Energ. Res. 36, 1105-1120 (2012).

4 Teng, X., Sun, C., Dai, J., Liu, H., Su, J. \& Li, F. Solution casting Nafion/ polytetrafluoroethylene membrane for vanadium redox flow battery application. Electrochim. Acta 88, 725-734 (2013).

5 Kim, J., Jeon, J. D. \& Kwak, S. Y. Nafion-based composite membrane with a permselective layered silicate layer for vanadium redox flow battery. Electrochem. Commun. 38, 68-70 (2014).

6 Yan, J., Huang, X., Moore, H. D., Wang, C. Y. \& Hickner, M. A. Transport properties and fuel cell performance of sulfonated poly(imide) proton exchange membranes. Int. J. Hydrogen Energ. 37, 6153-6160 (2012).

7 Mai, Z., Zhang, H., Li, X., Bi, C. \& Dai, H. Sulfonated poly(tetramethydiphenyl ether ether ketone) membranes for vanadium redox flow battery application. J. Power Sources 196, 482-487 (2011).

8 Zhang, Y. P., Li, J. C., Zhang, H., Zhang, S. \& Huang, X. D. Sulfonated polyimide membranes with different non-sulfonated diamines for vanadium redox battery applications. Electrochim. Acta 150, 114-122 (2014).

9 Semiz, L., Demirci Sankir, N. \& Sankir, M. Influence of the basic membrane properties of the disulfonated poly(arylene ether sulfone) copolymer membranes on the vanadium redox flow battery performance. J. Membrane Sci. 468, 209-215 (2014).

10 Li, J. C., Zhang, Y. P., Zhang, S., Huang, X. D. \& Wang, L. Novel sulfonated polyimide/ $\mathrm{ZrO}_{2}$ composite membrane as a separator of vanadium redox flow battery. Polym. Advan. Technol. 25, 1610-1615 (2014).

11 Zhang, Y. P., Li, J. C., Wang, L. \& Zhang, S. Sulfonated polyimide/AlOOH composite membranes with decreased vanadium permeability and increased stability for vanadium redox flow battery. J. Solid State Electr. 18, 3479-3490 (2014).

12 Li, J. C., Zhang, Y. P. \& Wang, L. Preparation and characterization of sulfonated polyimide/ $/ \mathrm{TiO}_{2}$ composite membrane for vanadium redox flow battery. J. Solid State Electr. 18, 729-737 (2013)

$13 \mathrm{Li}$, J. C., Zhang, Y. P., Zhang, S. \& Huang, X. D. Sulfonated polyimide/s-MoS 2 composite membrane with high proton selectivity and good stability for vanadium redox flow battery. J. Membrane Sci. 490, 179-189 (2015).

14 Chromik, A., dos Santos, A. R., Turek, T., Kunz, U., Häring, T. \& Kerres, J. Stability of acid-excess acid-base blend membranes in all-vanadium redox-flow batteries. J. Membrane Sci. 476, 148-155 (2015).

15 Smitha, B., Devi, D. A. \& Sridhar, S. Proton-conducting composite membranes of chitosan and sulfonated polysulfone for fuel cell application. Int. J. Hydrogen Energ. 33, 4138-4146 (2008).
16 Yue, M. Z., Zhang, Y. P. \& Wang, L. Sulfonated polyimide/chitosan composite membrane for vanadium redox flow battery: influence of the infiltration time with chitosan solution. Solid State Ionics 217, 6-12 (2012).

17 Yue, M. Z., Zhang, Y. P. \& Wang, L. Sulfonated polyimide/chitosan composite membrane for vanadium redox flow battery: membrane preparation, characterization, and single cell performance. J. Appl. Polymer Sci. 127, 4150-4159 (2013).

18 Wang, C., Young, L. S., Won, S. D., Rae, K. N., Lee, Y. M. \& Guiver, M. D. Proton-conducting membranes from poly(ether sulfone)s grafted with sulfoalkylamine. J. Membrane Sci. 427, 443-450 (2013).

19 Gao, N., Zhang, F., Zhang, S. \& Liu, J. Novel cardo poly(arylene ether sulfone)s with pendant sulfonated aliphatic side chains for proton exchange membranes. J. Membrane Sci. 372, 49-56 (2011).

20 Li, N., Lee, S. Y., Liu, Y. L., Lee, Y. M. \& Guiver, M. D. A new class of highly-conducting polymer electrolyte membranes: Aromatic ABA triblock copolymers. Energ. Environ. Sci. 5, 5346 (2012).

21 Chen, D. \& Hickner, M. A. V ${ }^{5+}$ degradation of sulfonated Radel membranes for vanadium redox flow batteries. Phys. Chem. Chem. Phys. 15, 11299-11305 (2013).

22 Yuan, Z., Li, X., Hu, J., Xu, W., Cao, J., \& Zhang, H. Degradation mechanism of sulfonated poly(ether ether ketone) (SPEEK) ion exchange membranes under vanadium flow battery medium. Phys. Chem. Chem. Phys. 16, 19841-19847 (2014).

23 Kim, S., Tighe, T. B., Schwenzer, B., Yan, J., Zhang, J., Liu, J., Yang, Z. \& Hickner, M. A. Chemical and mechanical degradation of sulfonated poly(sulfone) membranes in vanadium redox flow batteries. J. Appl. Electrochem. 41, 1201-1213 (2011).

24 Theresa, S. \& Maria, S. Membrane stability studies for vanadium redox cell applications. J. Appl. Electrochem. 34, 137-145 (2004).

25 Chen, D., Wang, S., Xiao, M., Han, D. \& Meng, Y. Sulfonated poly (fluorenyl ether ketone) membrane with embedded silica rich layer and enhanced proton selectivity for vanadium redox flow battery. J. Power Sources 195, 7701-7708 (2010).

26 Zheng, Q., Xing, F., Li, X., Liu, T., Lai, Q., Ning, G. \& Zhang, H. Dramatic performance gains of a novel circular vanadium flow battery. J. Power Sources 277, 104-109 (2015).

27 Gills, M., Carine, P., Gerard, G., Laurent, G., Sandrine, M. \& Jean-Luc, G. Ex situ hydrolytic degradation of sulfonated polyimide membranes for cells. Polymer 47, 5003-5011 (2006).

28 Seyed, F. H., Masoud, R., Mojgan, Z. \& Farhid, F. Development of bioactive fish gel/chitosan nanoparticles composite films with antimicrobial properties. Food Chem. 194, 1266-1274 (2016).

29 Fengxiang, Z., Huangmin, Z. \& Chao, Q. A dication cross-linked composite anionexchange membrane for all-vanadium flow battery applications. ChemSusChem 6 , 2290-2298 (2013).

30 Nanfang, W., Jingang, Y., Zhi, Z., Dong, F., Suqin, L. \& Younian, L. SPPEK/TPA composite membrane as a separator of vanadium redox flow battery. J. Membrane Sci. 437, 114-121 (2013).

31 Fujimoto, C., Kim, S., Stains, R., Wei, X., Li, L. \& Yang, Z. G. Vanadium redox flow battery efficiency and durability studies of sulfonated Diels Alder poly(phenylene)s. Electrochem. Commun. 20, 48-51 (2012).

32 Yuan, Z., Li, X., Duan, Y., Zhao, Y. \& Zhang, H. Application and degradation mechanism of polyoxadiazole based membrane for vanadium flow batteries. J. Power Sources 488 , 194-202 (2015).

33 Sun, J., Li, X., Xi, X., Lai, Q., Liu, T. \& Zhang, H. The transfer behavior of different ions across anion and cation exchange membranes under vanadium flow battery medium. J. Power Sources 271, 1-7 (2014).

34 Sun, J., Shi, D., Zhong, H., Li, X. \& Zhang, H. Investigations on the self-discharge process in vanadium flow battery. J. Power Sources 294, 562-568 (2015).

35 Agar, E., Benjamin, A., Dennison, C. R., Chen, D., Hickner, M. A. \& Kumbur, E. C. Reducing capacity fade in vanadium redox flow batteries by altering charging and discharging currents. J. Power Sources 246, 767-774 (2014).

36 Agar, E., Knehr, K. W., Chen, D., Hickner, M. A. \& Kumbur, E. C. Species transport mechanisms governing capacity loss in vanadium flow batteries: comparing Nafion and sulfonated Radel membranes. Electrochim. Acta 98, 66-74 (2013).

37 Sun, C., Chen, J., Zhang, H., Han, X. \& Luo, Q. Investigations on transfer of water and vanadium ions across Nafion membrane in an operating vanadium redox flow battery. J. Power Sources 195, 890-897 (2010).

38 Ding, C., Zhang, H., Li, X., Yao, C. \& Shi, D. Morphology and electrochemical properties of perfluorosulfonic acid ionomers for vanadium flow battery applications: effect of side-chain length. ChemSusChem 6, 1262-1269 (2013).

39 Ngamsai, K. \& Arpornwichanop, A. Analysis and measurement of the electrolyte imbalance in a vanadium redox flow battery. J. Power Sources 282, 534-543 (2015).

40 Chen, D., Hickner, M. A, Agar, E. \& Kumbur, E. C. Selective anion exchange membranes for high coulombic efficiency vanadium redox flow batteries. Electrochem. Commun. 26, 37-40 (2013).

41 Xi, J., Li, Z., Yu, L., Yin, B., Wang, L., Liu, L., Qiu, X. \& Chen, L. Effect of degree of sulfonation and casting solvent on sulfonated poly(ether ether ketone) membrane for vanadium redox flow battery. J. Power Sources 285, 195-204 (2015). 